\title{
MHV amplitudes at strong coupling and linearized TBA equations
}

\author{
Katsushi Ito, ${ }^{a}$ Yuji Satoh ${ }^{b}$ and Junji Suzuki ${ }^{c}$ \\ ${ }^{a}$ Department of Physics, Tokyo Institute of Technology, \\ Tokyo 152-8551, Japan \\ ${ }^{b}$ Institute of Physics, University of Tsukuba, \\ Ibaraki 305-8571, Japan \\ ${ }^{c}$ Department of Physics, Shizuoka University, \\ Shizuoka 422-8529, Japan \\ E-mail: ito@th.phys.titech.ac.jp, ysatoh@het.ph.tsukuba.ac.jp, \\ suzuki.junji@shizuoka.ac.jp
}

ABSTRACT: The maximally helicity violating (MHV) amplitudes of $\mathcal{N}=4$ super Yang-Mills theory at strong coupling are obtained by solving auxiliary thermodynamic Bethe ansatz (TBA) integral equations. We consider a limit where the TBA equations are linearized for large chemical potentials and masses therein. By solving the linearized equations, we derive analytic expansions of the 6-point MHV amplitudes in terms of the ratio of the chemical potential $A$ and the mass $M$. The expansions are valid up to corrections exponentially small in $A$ or inversely proportional to powers of $A$. The analytic expansions describe the amplitudes for small conformal cross-ratios of the particle momenta in a standard basis, and interpolate the amplitudes with equal cross-ratios and those in soft/collinear limits. The leading power corrections are also obtained analytically. We compare the 6-point rescaled remainder functions at strong coupling and at 2 loops for the above kinematics. They are rather different, in contrast to other kinematic regions discussed in the literature where they are found to be similar to each other.

KEYwords: AdS-CFT Correspondence, Bethe Ansatz, Integrable Field Theories, Scattering Amplitudes

ARXiv EPrint: 1805.07556 


\section{Contents}

1 Introduction 1

2 Scattering amplitudes at strong coupling $\quad 3$

2.1 TBA equations for MHV amplitudes 4

2.2 Area, remainder function and cross-ratios 5

$\begin{array}{lll}2.3 & \text { Limits of the TBA system } & 7\end{array}$

2.4 6-particle amplitudes 8

3 Linearized TBA equation $\quad 10$

$\begin{array}{lll}3.1 & \text { Linearization } & 10\end{array}$

$\begin{array}{ll}3.2 & \text { Analysis of the linearized TBA } \\ 3.3\end{array}$

$\begin{array}{lll}3.3 & \mathcal{O}\left(L^{-2}\right) \text { corrections } & 15\end{array}$

$\begin{array}{lll}3.4 & \text { Free energy } & 19\end{array}$

$\begin{array}{ll}3.5 & \text { Leading order expansions in } 1 / L \\ \end{array}$

4 Numerical analysis $\quad 22$

4.1 Dependence on $L \quad 22$

4.2 Dependence on $m / a \quad 24$

5 Analytic expansion of 6-point remainder function $\mathbf{2 5}$

5.1 Cross-ratios 25

5.2 Expansion of 6-point remainder function 28

$\begin{array}{lll}5.3 & \text { Comparison with 2-loop results } & 29\end{array}$

6 Conclusions $\quad 30$

A Evaluation of $\tilde{\epsilon}$ from direct integrals $\quad 32$

B Estimation of $\epsilon^{\prime}(B) \quad 33$

\section{Introduction}

Developments in the study of the gauge-string duality and perturbative gauge theory have merged into a deep understanding of the four-dimensional maximally supersymmetric YangMills theory $(\mathcal{N}=4 \mathrm{SYM})$. It is now possible to address its dynamics even at finite coupling based on the underlying integrability in the planar limit [1]: one can find the spectrum of single-trace operators [2-4], and a formulation has been given [5] to obtain the scattering amplitudes, or equivalently [6-10], the expectation values of null-polygonal Wilson loops. 
Such integrability-based approaches have also been extended to the correlation functions of single-trace operators $[11,12]$.

Focusing on the maximally helicity violating (MHV) amplitudes, the form of the $n$ point amplitudes is almost fixed by the Bern-Dixon-Smirnov (BDS) expression [13] due to the anomalous conformal Ward identity $[9,10]$. The evaluation of the full amplitudes thus reduces to finding its remainder which exists for $n \geq 6$ beyond one loop [14-16]. A notable fact to this end is that the analytic structure of the amplitudes is well controlled by the transcendentality and the associated symbol of loop integrals. This enables us to "bootstrap" the amplitudes. The complete and concise expression of the 6-point amplitudes at 2 loops has been obtained in this way [17], in agreement with the direct computation [18]. The bootstrap method has been extended up to 5 loops for $n=6$ [19-21], and up to 2 loops for $n=7$ [22]. For the restricted kinematics where the momenta of particles are two dimensional, analytic results are also given in [23, 24].

At strong coupling, the MHV amplitudes are obtained [6] by evaluating the area of the minimal surfaces in the five-dimensional anti-de Sitter space $\left(\mathrm{AdS}_{5}\right)$ whose boundary ends on the corresponding null-polygonal Wilson loops at the boundary of $\mathrm{AdS}_{5}$. Although only a few exact solutions are known for such null-polygonal minimal surfaces [6, 25-28], one can evaluate their area by solving auxiliary integral equations [29-32]. These integral equations take the form of the thermodynamic Bethe ansatz (TBA) equations, which appear in the analysis of finite-size effects in integrable models.

The equations for $n=6$ are indeed identified [30] with the TBA equations of the $\mathbb{Z}_{4^{-}}$ integrable model [33]. When the momenta of particles are restricted to three-dimensional spacetime, the minimal surfaces are embedded in the $\mathrm{AdS}_{4}$ subspace. In this case, the integral equations for the $n$-point amplitudes are identified [32] with the TBA equations of the $s u(n-4)_{4} / u(1)^{n-5}$ homogeneous sine-Gordon (HSG) model [34]. For the twodimensional kinematics for which the corresponding minimal surfaces are embedded in $\mathrm{AdS}_{3}$, they are identified [32] with the TBA equations of the $s u(n / 2-2)_{2} / u(1)^{n / 2-3}$ HSG model. The integrable models in the general $\mathrm{AdS}_{5}$ case have not been identified yet for $n \geq 7$, in particular due to the unusual nature of the integral equations.

The integral equations for the amplitudes can be solved numerically by iteration. In addition, the equations can be simplified by taking limits of the parameters therein. For example, when the "mass" parameters are large, the integrable model reduces to a free massive theory in the infrared regime. The solution to the TBA equations is then expressed by iterative multiple integrals [35]. On the SYM side, the cross-ratios formed by particle momenta may become large or small in this limit. This includes the collinear limit for which the OPE/flux-tube expansions of the amplitudes are formulated at finite coupling [5, 36]. On the other hand, in the strict limit of vanishing masses (the ultraviolet regime), the TBA equations are solved analytically [35]. From the point of view of SYM, it is the limit where the corresponding Wilson loops form regular polygons. Around this regularpolygonal limit, the amplitudes are expanded analytically by small masses for $n=6$ in the $\mathrm{AdS}_{5}$ case [37, 38], and for general $n$ in the $\mathrm{AdS}_{4}$ [39] and the $\mathrm{AdS}_{3}[40,41]$ case.

In this paper, we consider another limit of the TBA equations for the amplitudes, where the "chemical potentials" as well as the masses are large and hence the equations 
are linearized. The corrections in the linearization are exponentially small in the chemical potentials, or suppressed by their powers as understood by the Sommerfeld type argument [42]. In the case of the 6-point amplitudes, which we discuss concretely below, the linearized equations are solved following [43] as expansions in the ratio of the mass $M$ and the chemical potential $A$ to any order. The power corrections are analyzed by extending the analysis in [43], and we show that the leading power corrections are analytically evaluated. The results are checked against the numerical solutions, to be found in agreement. These analyses of the corrections assure that the linearization gives a controlled approximation of the original TBA equations for large $A$ and $M$.

Applying the solution of the linearized TBA equations, we derive analytic expansions of the 6-point MHV amplitudes to any order. As the parameters are varied, the cross ratios of the particle momenta in a standard basis are kept small and change from the equal value in the UV regime to those for the soft/collinear limits in the IR regime. The amplitudes are well described by the expansion over the corresponding kinematic region from the UV regime to the IR regime. Our results thus provide another concrete example where focusing on the strong coupling enables us to explicitly evaluate the MHV amplitudes. Since the collinear limit can be realized as the IR end point in the present case, it is of interest to consider implications of our expansion to the OPE/flux-tube expansion at finite coupling.

Along the above trajectory of the cross-ratios, we also compare the 6-point remainder functions at strong coupling and at 2 loops which are rescaled/normalized by their UV and IR values. They turn out to be rather different in contrast to the cases where similarities are found between the strong-coupling results and the perturbative results [19, 20, 38-41, 44]. This implies that the kinematic region described by our expansion provides a probe to study structural differences of the strong-coupling and the perturbative results.

This paper is organized as follows: in section 2, we review the MHV amplitudes at strong coupling and the associated TBA system. In section 3, we solve the linearized TBA equations for the 6-point amplitudes, and analyze the corrections in the linearization inversely proportial to powers of $A$. In section 4 , we check the results in section 3 against numerical solutions. In section 5, we derive analytic expansions of the 6-point MHV amplitudes, and compare the rescaled remainder functions at strong coupling and at 2 loops. We conclude with a summary and discussion in section 6 . Two appendices are also attached. In appendix A, we evaluate one of the pseudo energies in a different way from the main text by direct integration. This shows explicitly how the fractional powers of the spectral parameter appear from the summation over its integral powers, in accordance with the periodicity which is required from the algebraic equations (Y-system) associated with the TBA equations. In appendix B, we estimate the derivative of a pseudo energy.

\section{Scattering amplitudes at strong coupling}

Let us consider the MHV amplitude $\mathcal{M}$ of $\mathcal{N}=4 \mathrm{SYM}$ in the planar limit. This is equivalent/dual to the expectation value of the null-polygonal Wilson loops whose edges correspond to the momenta of scattering particles [6-10]. After the tree amplitude is factored out, the remaining scalar part at strong coupling is thus evaluated by the area $\mathcal{A}$ 
of the minimal surfaces in $\mathrm{AdS}_{5}$ ending on the null-polygonal Wilson loops at the boundary of $\mathrm{AdS}_{5}$ [6]. Schematically,

$$
\mathcal{M} \sim e^{-\frac{\sqrt{\lambda}}{2 \pi} \mathcal{A}},
$$

where $\lambda \gg 1$ is the 't Hooft coupling. Since $\mathcal{N}=4 \mathrm{SYM}$ is conformal, natural kinematical variables to express amplitudes are the cross-ratios formed by momenta of particles.

\subsection{TBA equations for MHV amplitudes}

Although only a few solutions are known in closed forms for such null-polygonal minimal surfaces [6, 25-28], one can evaluate their area by solving auxiliary integral equations [2932]. For $n$-particle amplitudes, they take the form [31],

$$
\begin{aligned}
& \log Y_{2, s}(\theta)=-\sqrt{2} M_{s} \cosh \theta-K_{2} * \mathcal{L}_{1 s}-K_{1} * \mathcal{L}_{2 s}, \\
& \log Y_{1, s}(\theta)=-M_{s} \cosh \theta-C_{s}-\frac{1}{2} K_{2} * \mathcal{L}_{2 s}-K_{1} * \mathcal{L}_{1 s}-\frac{1}{2} K_{3} * \mathcal{L}_{3 s}, \\
& \log Y_{3, s}(\theta)=-M_{s} \cosh \theta+C_{s}-\frac{1}{2} K_{2} * \mathcal{L}_{2 s}-K_{1} * \mathcal{L}_{1 s}+\frac{1}{2} K_{3} * \mathcal{L}_{3 s},
\end{aligned}
$$

where $M_{s}, C_{s}$ are constants, $s=1, \ldots, n-5$, and

$$
\begin{aligned}
\mathcal{L}_{1 s} & =\log \frac{\left(1+Y_{1, s}\right)\left(1+Y_{3, s}\right)}{\left(1+Y_{2, s-1}\right)\left(1+Y_{2, s+1}\right)}, \quad \mathcal{L}_{3 s}=\log \frac{\left(1+Y_{1, s-1}\right)\left(1+Y_{3, s+1}\right)}{\left(1+Y_{1, s+1}\right)\left(1+Y_{3, s-1}\right)}, \\
\mathcal{L}_{2 s} & =\log \frac{\left(1+Y_{2, s}\right)^{2}}{\left(1+Y_{1, s-1}\right)\left(1+Y_{1, s+1}\right)\left(1+Y_{3, s-1}\right)\left(1+Y_{3, s+1}\right)} .
\end{aligned}
$$

The Y-functions, $Y_{a, s}$, are defined through the Stokes data of the auxiliary linear problem associated with the string equations of motion. We have also denoted by $*$ the convolution $f * g(\theta):=\int_{-\infty}^{\infty} \frac{d \theta^{\prime}}{2 \pi} f\left(\theta-\theta^{\prime}\right) g\left(\theta^{\prime}\right)$, with the kernels,

$$
K_{1}(\theta)=\frac{1}{\cosh \theta}, \quad K_{2}(\theta)=\frac{2 \sqrt{2} \cosh \theta}{\cosh 2 \theta}, \quad K_{3}(\theta)=2 i \tanh 2 \theta .
$$

Numerically, these equations are solved by iteration where the initial values of $\log Y_{a, s}$ are approximated by the "driving terms", i.e. the terms not involving convolutions in (2.1).

Though $M_{s}$ are assumed to be real and positive in (2.1), they are complex in general as $M_{s}=\left|M_{s}\right| e^{i \varphi_{s}}$. For small $\varphi_{s}$, the equations (2.1) keep the same form but with $M_{s} \rightarrow\left|M_{s}\right|$,

$$
Y_{a, s}(\theta) \rightarrow Y_{a, s}\left(\theta+i \varphi_{s}\right), \quad K_{s, s^{\prime}}^{a, a^{\prime}}\left(\theta-\theta^{\prime}\right) \rightarrow K_{s, s^{\prime}}^{a, a^{\prime}}\left(\theta-\theta^{\prime}+i \varphi_{s}-i \varphi_{s^{\prime}}\right),
$$

where $K_{s, s^{\prime}}^{a, a^{\prime}}$ is the kernel $K_{j}$ for the convolution involving $Y_{a, s}$ and $Y_{a^{\prime}, s^{\prime}}$. When $\left|\varphi_{s}-\varphi_{s+1}\right|$ exceeds $\pi / 4$, extra terms appear as the integrals pick up the poles of the integrands.

This formulation covers signatures of the four-dimensional spacetime other than the usual $(3,1)$ of $\mathbb{R}^{3,1}$. By the reality condition of the minimal surfaces, $C_{s}$ are required to be purely imaginary for the $(3,1)$ and $(1,3)$ signatures, whereas $C_{s}$ are real for the $(2,2)$ signature. The number of $\left|M_{s}\right|, \varphi_{s}, C_{s}$, i.e. $3(n-5)$, matches the number of the independent cross-ratios formed by the momenta of the scattering particles. 
The TBA equations (2.1) can be converted to a set of algebraic equations, called the Y-system,

$$
\frac{Y_{a, s}^{-} Y_{4-a, s}^{+}}{Y_{a+1, s} Y_{a-1, s}}=\frac{\left(1+Y_{a, s+1}\right)\left(1+Y_{4-a, s-1}\right)}{\left(1+Y_{a+1, s}\right)\left(1+Y_{a-1, s}\right)}
$$

where $a=1,2,3 ; s=1, \ldots, n-5$; and $Y_{a, 0}=Y_{a, n-4}=0$ and $Y_{0, s}=Y_{4, s}=\infty$. The superscripts \pm stand for the shift of the argument,

$$
f^{ \pm}(\theta):=f^{[ \pm 1]}(\theta), \quad f^{[k]}(\theta):=f\left(\theta+\frac{k}{4} \pi i\right) .
$$

From this Y-system, the Y-functions turn out to have the (quasi-)periodicity [45-48],

$$
Y_{a, s}^{[n]}(\theta)=\left\{\begin{array}{ll}
Y_{a, n-4-s}(\theta) & (s: \text { odd }) \\
Y_{4-a, n-4-s}(\theta) & (s: \text { even })
\end{array} .\right.
$$

The Y-system can also be used to obtain the Y-functions with large imaginary shift of the argument, for which the TBA equations (2.1) are modified due to the pole contributions. For a review of Y-systems, see for example [49].

The integral equations of the type (2.1) appear in the analysis of finite-size effects in integrable models, and are called the thermodynamic Bethe ansatz equations [35]. As mentioned in the introduction, the equations (2.1) are identified [30] with the TBA equations of the $\mathbb{Z}_{4}$-integrable model [33] for $n=6$. When $Y_{1, s}=Y_{3, s}$ and hence $C_{s}=0$, the minimal surfaces are embedded in $\mathrm{AdS}_{4}$, which describe the scattering of the special kinematics with three-dimensional momenta. In this case, (2.1) are identified [32] with the TBA equations of the $s u(n-4)_{4} / u(1)^{n-5}$ homogeneous sine-Gordon model [34]. By imposing further constraints, the minimal surfaces are embedded in $\mathrm{AdS}_{3}$, which describe the scattering for the two-dimensional kinematics. In this case, $n$ is even because of the momentum conservation, and (2.1) become the TBA equations of the $s u(n / 2-2)_{2} / u(1)^{n / 2-3}$ HSG model [32]. In the integrable models, the logarithms of the Y-functions $Y_{a, s}$ are pseudo energies, $M_{s}$ are masses of particles (measured in the unit of the inverse system size $1 / L$ ), and $C_{s}$ are chemical potentials. The corresponding integrable models in the $\mathrm{AdS}_{5}$ case have not been identified yet for $n \geq 7$. From the TBA point of view, what is unusual in (2.1) in this case is that the kernel $K_{3}(\theta)$ does not decay for large $|\theta|$.

\subsection{Area, remainder function and cross-ratios}

After a proper regularization, the area is expressed for $n \notin 4 \mathbb{Z}$ as

$$
\mathcal{A}=A_{\text {div }}+A_{\text {BDS-like }}+A_{\text {periods }}+A_{\text {free }},
$$

up to a constant. Here, $A_{\text {div }}$ is a divergent term, and $A_{\mathrm{BDS}-\text { like }}$ is the term which satisfies the anomalous conformal Ward identity [9, 10], as the Bern-Dixon-Smirnov (BDS) expression [13]. The third term $A_{\text {periods }}$ comes from period integrals associated with the underlying auxiliary linear problem or the Hitchin system, and is expressed by the mass parameters $M_{s}=\left|M_{s}\right| e^{i \varphi_{s}}$. The explicit forms of $A_{\mathrm{div}}, A_{\mathrm{BDS}-\text { like }}$ and $A_{\text {periods }}$ are found in [29-31]. The last term $A_{\text {free }}$ is obtained from the solution to the TBA equations (2.1). 
It coincides with the free energy of the corresponding integrable model (when it exits). Explicitly,

$$
A_{\text {free }}=\sum_{s} \int_{-\infty}^{\infty} \frac{d \theta}{2 \pi}\left|M_{s}\right| \cosh \theta \cdot \log \left[\left(1+Y_{1, s}\left(\theta_{s}\right)\right)\left(1+Y_{3, s}\left(\theta_{s}\right)\right)\left(1+Y_{2, s}\left(\theta_{s}\right)\right)^{\sqrt{2}}\right],
$$

with $\theta_{s}:=\theta+i \varphi_{s}$. For $n \in 4 \mathbb{Z}$, the expression of the area may be obtained by taking an appropriate limit from $n \notin 4 \mathbb{Z}$. Given the formula (2.6), the non-trivial part of the area/strong-coupling amplitudes reduces to $A_{\text {free }}$.

Since the structure of the MHV amplitudes is almost captured by the BDS expression, it may be sufficient to consider its remainder [14-16],

$$
R:=-\left(\mathcal{A}-A_{\mathrm{div}}-A_{\mathrm{BDS}}\right)=\Delta A_{\mathrm{BDS}}-A_{\text {periods }}-A_{\text {free }},
$$

where $A_{\mathrm{BDS}}$ is the (finite part of the) BDS expression, and $\Delta A_{\mathrm{BDS}}:=A_{\mathrm{BDS}}-A_{\mathrm{BDS}-\text { like }}$. This remainder functions is conformally invariant and is a function of the cross-ratios of the particle momenta $p_{j}^{\mu}$,

$$
\chi_{i j k l}:=\frac{x_{i j}^{2} x_{k l}^{2}}{x_{i k}^{2} x_{j l}^{2}}, \quad x_{i j}^{\mu}:=x_{i}^{\mu}-x_{j}^{\mu}, \quad p_{j}^{\mu}=x_{j}^{\mu}-x_{j+1}^{\mu} .
$$

The momenta form null polygons corresponding to the dual Wilson loops due to the momentum conservation. The subscript of the cusp points $x_{j}$ is hence understood modulo $n$. These cross-ratios are expressed by the Y-functions at special values of the argument, e.g.

$$
\chi_{k,-k,-k-1, k-1}=U_{2 k-2}^{\langle 0\rangle}, \quad \chi_{k+1,-k,-k-1, k}=U_{2 k-1}^{\langle 1\rangle},
$$

where we have defined

$$
U_{s}(\theta):=1+\frac{1}{Y_{2, s}(\theta)}, \quad f^{\langle k\rangle}:=f^{[k]}(0)=f\left(\frac{k}{4} \pi i\right) .
$$

A shift of $\theta$ induces a cyclic shift of the cusp points,

$$
U_{2 k-2}^{\langle 2 r\rangle}=\chi_{k+r,-k+r,-k-1+r, k-1+r}, \quad U_{2 k-1}^{\langle 2 r+1\rangle}=\chi_{k+1+r,-k+r,-k-1+r, k+r} .
$$

The product of these cross-ratios yields generic ones.

A useful parametrization of the cross-ratios is given by the coordinates $\left(\tau_{s}, \sigma_{s}, \phi_{s}\right)$ associated with the symmetries of (parts of) the null-polygons $[36,50]$. They are directly related to the Y-functions,

$$
\widehat{Y}_{1, s}(0)=e^{\phi_{s}-\sigma_{s}-\tau_{s}}, \quad \widehat{Y}_{2, s}(0)=e^{-2 \tau_{s}}, \quad \widehat{Y}_{3, s}(0)=e^{-\phi_{s}-\sigma_{s}-\tau_{s}},
$$

where $s=1, \ldots, n-5$, and

$$
\widehat{Y}_{a, s}(\theta):=\left\{\begin{array}{l}
Y_{a, s}(\theta)(a+s: \text { even }) \\
Y_{a, s}^{-}(\theta)(a+s: \text { odd })
\end{array} .\right.
$$

In the OPE approach $[5,36]$, these are used to compute the finite coupling amplitudes, which are not restricted to the MHV case. The amplitudes there are expanded by the contributions from the flux-tube excitations around the multi-collinear limit $\tau_{s} \rightarrow \infty$. Re-summing over such series recovers the TBA equations at strong coupling [51, 52]. The explicit relation of $\left(\tau_{s}, \sigma_{s}, \phi_{s}\right)$ and the cross-ratios $\chi_{i j k l}$ in (2.8) is found by using (2.9), (2.10) and the Y-system (2.4). 


\subsection{Limits of the TBA system}

The analysis of the TBA equations (2.1) can be simplified by taking the limits of the parameters therein.

As for the mass parameters $\left|M_{s}\right|$, there are two simple limits where $Y_{a, s}$ and $A_{\text {free }}$ are analytically evaluated.

Large mass/IR limit: one is the limit where all $\left|M_{s}\right| \gg 1$. This is the low temperature/IR limit where the integrable models reduce to free massive theories. The Y-functions are given by the driving terms, and the free energy trivially vanishes. From the point of view of SYM, it is the limit where cross-ratios can be large or small. Around this limit, the Y-functions and the free energy are expanded by iterative multiple integrals [35]. By analytic continuation, this limit is also connected to the Regge limit [53]. Further adjusting the phases $\varphi_{s}$ yields the above-mentioned multi-collinear limit.

Small mass/UV limit: the other is the limit where all $\left|M_{s}\right| \ll 1$. This is the high temperature/UV limit where the integrable models reduce to conformal field theories (CFTs). From the point of view of SYM, it is the limit where the dual null polygonal Wilson loops become $\mathbb{Z}_{n}$-symmetric (regular-polygonal). By the standard method on the TBA system [35], or from the Y-system (2.4) with the $\theta$-dependence dropped, the Y-functions and the free energy are evaluated explicitly in the strict limit of vanishing masses. We supply a concrete example for $n=6$ shortly in subsection 2.4. Around this limit, the free energy is expanded by conformal perturbation [35].

For the hexagonal minimal surfaces in $\mathrm{AdS}_{5}$, the Y-functions and hence the amplitudes are expanded around the small mass limit based on the quantum Wronskian relation [38] following an earlier work [37]. For the minimal surfaces in $\mathrm{AdS}_{3}$ [40, 41] and $\mathrm{AdS}_{4}$ [39], the small-mass expansions of the Y-functions and the amplitudes are derived based on the relation [54-56] between the $g$ - and Y-functions using auxiliary boundary conformal perturbation. The expansions by the conformal perturbation are in terms of the couplings of the relevant operators to the CFT in the UV limit. They are expressed by the mass (and other) parameters of the TBA system via the mass-coupling relation. For the hexagonal minimal surfaces in $\mathrm{AdS}_{5}$, the corresponding $\mathbb{Z}_{4}$ integrable model has a single mass parameter, and the relevant exact mass-coupling relation is given in [57] based on the work [43]. When the models have multi-scales, it is in general difficult to obtain such relations. In [58, 59], for the analysis of multi-scale integrable models, the exact mass-coupling relation is obtained in the $n=10$ case for the $\mathrm{AdS}_{3}$ minimal surfaces. (For $\mathrm{AdS}_{3}$, the TBA system has multi-scales for $n \geq 10$.)

Phases: as for the phases $\varphi_{s}$, they appear as the imaginary shifts of $Y_{a, s}(\theta)$ as in (2.3). Their change induces the "wall-crossing" phenomenon, or the change of the form of the TBA equations (2.1). They are restricted to a finite range because of the periodicity of the Y-functions (2.5).

Large chemical potentials: as for the last parameters $C_{s}$, it follows from the TBA equations that

$$
\log \frac{Y_{3, s}}{Y_{1, s}}=2 C_{s}+K_{3} * \mathcal{L}_{3 s} \sim 2 C_{s},
$$


where we have used a rough estimate for large $C_{s}$ and $\left|M_{s}\right|$ such that $\log Y_{a, s}$ are approximated in (2.1) by the driving terms. From (2.11), one finds that $C_{s}$ are directly related to $\phi_{s}$ (Lorentz boost coordinates) in the cross-ratios. In the following, we consider the case of $n=6$ and demonstrate that the large potentials $C_{s}$ indeed provide another useful limit in analyzing the TBA system for the amplitudes. Precisely, we combine two limits, large mass and chemical potential, which has not yet been considered so far.

\subsection{6-particle amplitudes}

When the number of the particles $n=6$, there are only three non-trivial Y-functions $Y_{1,1}, Y_{2,1}, Y_{3,1}$. Thus, $\mathcal{L}_{3 s}$ in $(2.1)$ is vanishing, and the ratio $Y_{3,1} / Y_{1,1}$ is a constant. The TBA equations then reduce to

$$
\begin{aligned}
& \varepsilon(\theta)=-A+M \cosh \theta+K_{1} * \log \left(1+\mu^{-2} e^{-\varepsilon}\right)\left(1+e^{-\varepsilon}\right)+K_{2} * \log \left(1+e^{-\tilde{\varepsilon}}\right), \\
& \tilde{\varepsilon}(\theta)=\sqrt{2} M \cosh \theta+K_{2} * \log \left(1+\mu^{-2} e^{-\varepsilon}\right)\left(1+e^{-\varepsilon}\right)+2 K_{1} * \log \left(1+e^{-\tilde{\varepsilon}}\right)
\end{aligned}
$$

where we have set

$$
\log Y_{1}(\theta+i \varphi)=-\varepsilon(\theta)-2 A, \quad \log Y_{3}(\theta+i \varphi)=:-\varepsilon(\theta), \quad \log Y_{2}(\theta+i \varphi)=:-\tilde{\varepsilon}(\theta) .
$$

with

$$
Y_{a}(\theta):=Y_{a, 1}(\theta), \quad M:=\left|M_{1}\right|, \quad \mu:=e^{A}, \quad A:=C_{1}, \quad \varphi:=\varphi_{1} .
$$

From now on, we assume $|\varphi|<\pi / 4$, unless otherwise stated, so that the TBA equations take the form as in (2.1) without the pole contributions. The Y-system (2.4) for $n=6$ reads

$$
Y_{1}^{+} Y_{3}^{-}=\frac{Y_{2}}{1+Y_{2}}, \quad Y_{2}^{+} Y_{2}^{-}=\frac{Y_{1} Y_{3}}{\left(1+Y_{1}\right)\left(1+Y_{3}\right)} .
$$

Then the equation for $Y_{3}^{+} Y_{1}^{-}$is equivalent to the first one because $Y_{3} / Y_{1}=\mu^{2}$. From (2.5) or (2.14), it follows that

$$
Y_{a}^{[6]}=Y_{a} .
$$

The components of the area or the remainder function in (2.7) are

$$
A_{\text {free }}=\int_{-\infty}^{\infty} \frac{d \theta}{2 \pi} M \cosh \theta \times \log \left(1+\mu^{-2} e^{-\varepsilon(\theta)}\right)\left(1+e^{-\varepsilon(\theta)}\right)\left(1+e^{-\tilde{\varepsilon}(\theta)}\right)^{\sqrt{2}},
$$

and

$$
A_{\text {period }}=\frac{1}{4} M^{2}, \quad \Delta A_{\mathrm{BDS}}=-\frac{1}{4} \sum_{j=1}^{3} \mathrm{Li}_{2}\left(1-\frac{1}{u_{j}}\right) .
$$

For the 6-point amplitudes, there are three independent cross-ratios. A standard basis for them is

$$
u_{1}:=\chi_{2653}=\frac{1}{U^{\langle 3\rangle}}, \quad u_{2}:=\chi_{3164}=\frac{1}{U^{\langle 5\rangle}}, \quad u_{3}:=\chi_{4215}=\frac{1}{U^{\langle 1\rangle}},
$$

with $U:=U_{1}$. They are also parametrized by the variables of the type (2.11),

$$
Y_{1}^{\langle 0\rangle}=e^{\phi-\sigma-\tau}, \quad Y_{2}^{\langle-1\rangle}=e^{-2 \tau}, \quad Y_{3}^{\langle 0\rangle}=e^{-\phi-\sigma-\tau},
$$


with $(\tau, \sigma, \phi):=\left(\tau_{1}, \sigma_{1}, \phi_{1}\right)$. From the periodicity $(2.15)$, one has $Y_{2}^{\langle-1\rangle}=Y_{2}^{\langle 5\rangle}$, and $u_{2}$ is readily related to $\tau$. Furthermore, $u_{1,3}$ are related to $(\tau, \sigma, \phi)$ through

$$
\begin{aligned}
Y_{2}^{\langle 1\rangle} & =\frac{Y_{1}^{\langle 0\rangle} Y_{3}^{\langle 0\rangle}}{\left(1+Y_{1}^{\langle 0\rangle}\right)\left(1+Y_{3}^{\langle 0\rangle}\right) Y_{2}^{\langle-1\rangle}}, \\
Y_{2}^{\langle 3\rangle} & =\frac{Y_{2}^{\langle-1\rangle}}{\left(Y_{2}^{\langle-1\rangle}+Y_{1}^{\langle 0\rangle}\left(1+Y_{2}^{\langle-1\rangle}\right)\right)\left(Y_{2}^{\langle-1\rangle}+Y_{3}^{\langle 0\rangle}\left(1+Y_{2}^{\langle-1\rangle}\right)\right)},
\end{aligned}
$$

which follows from the Y-system. We thus find [5], ${ }^{1}$

$$
\frac{1}{u_{2}}=1+e^{2 \tau}, \quad \frac{u_{1}}{u_{2} u_{3}}=e^{2 \sigma+2 \tau}, \quad \frac{1}{u_{3}}=1+e^{-2 \tau}\left(1+e^{\tau+\sigma+\phi}\right)\left(1+e^{\tau+\sigma-\phi}\right) .
$$

UV and IR limits: for reference for the discussion below, we summarize the behavior of the pseudo energies, the free energy and the remainder function in the UV and the IR limit. First, in the UV limit $M \rightarrow 0$ with $A$ and $\varphi$ fixed,

$$
\begin{aligned}
e^{\varepsilon} & \rightarrow e^{\varepsilon_{\mathrm{UV}}}:=\frac{1}{\mu}\left(\mu^{1 / 3}+\mu^{-1 / 3}\right), \quad e^{\tilde{\varepsilon}} \rightarrow e^{\tilde{\varepsilon}_{\mathrm{UV}}}:=1+\mu^{2 / 3}+\mu^{-2 / 3}, \\
A_{\text {free }} \rightarrow A_{\text {free }, \mathrm{UV}} & :=\frac{\pi}{6} c+\frac{A^{2}}{3 \pi},
\end{aligned}
$$

where $c=1$ is the central charge of the CFT in the UV limit of the underlying $\mathbb{Z}_{4}$ integrable model. These imply all $u_{j} \rightarrow \hat{u}_{\mathrm{UV}}:=1 /\left(\mu^{1 / 3}+\mu^{-1 / 3}\right)^{2}$, and

$$
R \rightarrow R_{6, \mathrm{UV}}:=-\frac{3}{4} \operatorname{Li}_{2}\left(1-\frac{1}{\hat{u}_{\mathrm{UV}}}\right)-\frac{\pi}{6}-\frac{A^{2}}{3 \pi} .
$$

As mentioned above, the boundary of the minimal surface becomes regular-polygonal in this limit.

On the other hand, in the IR limit $M \rightarrow \infty$ with $A$ and $\varphi$ fixed,

$$
\begin{aligned}
\varepsilon & \rightarrow \varepsilon_{\mathrm{IR}}:=-A+M \cosh \theta, \quad \tilde{\varepsilon} \rightarrow \tilde{\varepsilon}_{\mathrm{IR}}:=\sqrt{2} M \cosh \theta, \\
A_{\text {free }} & \rightarrow A_{\text {free }, \mathrm{IR}}:=0 .
\end{aligned}
$$

Again from the Y-system, these imply that two of $u_{j} \rightarrow 0$ and the other $u_{k} \rightarrow 1$ for generic $\varphi$, and

$$
R \rightarrow R_{6, \mathrm{IR}}:=\frac{\pi^{2}}{12} .
$$

This is the soft limit, e.g. where $u_{1} \sim 1, u_{2,3} \sim 0$ and both $\tau, \sigma \gg 1$ for $-\pi / 4<\varphi<0$ [30]. Sending $\varphi \rightarrow-\pi / 4$, one can achieve the collinear limit where $u_{2} \sim 0, u_{1}+u_{3} \sim 1$ and $\tau \gg 1$ with $\sigma$ fixed. This is the regime of the OPE expansion $[5,36]$. By changing $\varphi$, the roles of $u_{j}$ are permuted. For higher order terms in the UV and the IR limit, we refer to $[37,38]$.

Figure 1 illustrates these limits in the space of the three cross-ratios $\left(u_{1}, u_{2}, u_{3}\right)$. The red straight line represents $u_{1}=u_{2}=u_{3}$ corresponding to the UV limit. The triangle

\footnotetext{
${ }^{1}$ Comparing with the parametrization in $[36], e^{-\tau_{\text {there }}}=e^{\tau}\left(-1+\sqrt{1+e^{-2 \tau}}\right), e^{-\sigma_{\text {there }}}=e^{-\sigma} \sqrt{1+e^{-2 \tau}}$.
} 


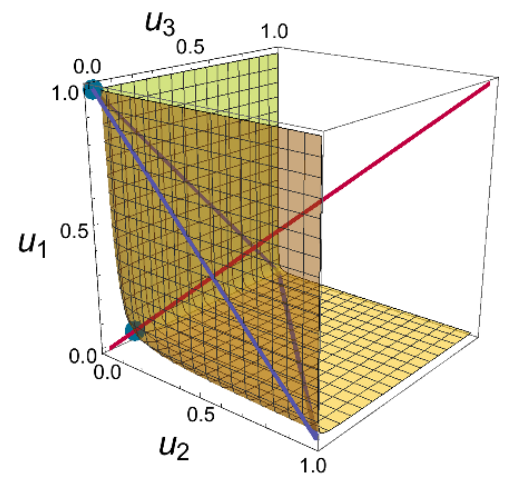

Figure 1. Cross-ratios and various limits. The red straight line corresponds to the UV limit. The triangle with vertices $\left(u_{1}, u_{2}, u_{3}\right)=(1,0,0),(0,1,0),(0,0,1)$ corresponds to the IR limit. In particular, its sides and vertices correspond to the collinear and the soft limit, respectively.

represents the IR limit. Its sides correspond to the collinear limits, whereas its vertices $\left(u_{1}, u_{2}, u_{3}\right)=(1,0,0),(0,1,0),(0,0,1)$ to the soft limit. The yellow surface represents $u_{1} u_{2} u_{3}=e^{-2 A}$, which is discussed in section 5. Based on the linearized TBA equations in the next section, the expansions of $Y_{a}, A_{\text {free }}$ and $R$ for large $A$ and $M$ are derived there. They interpolate the kinematic points in the UV and the IR limit, e.g. which are marked by the green blobs for generic $-\pi / 4<\varphi<0$. When the phase is adjusted, e.g. as $\varphi \rightarrow-\pi / 4$, the point in the IR limit moves onto a side of the triangle away from its vertices.

\section{Linearized TBA equation}

In this section, we consider the TBA equations for the 6-point amplitudes (2.12) for large $A$ and $M$ where the equations are linearized. We first introduce the linearlized TBA equations in subsection 3.1 and summarize the solution to the linearized equations following [43] in subsection 3.2. There are several types of the corrections in the linearization. One is exponentially small in $A$ (as in (3.2)), another is expected to be exponentially small in $A$ (as in $\Delta_{2}$ in (3.5)), and the other is suppressed by the powers of $A$ (as in $\Delta_{1}$ in (3.5)) due to the Sommerfeld type argument [42]. The exponential behavior of the second type is confirmed by numerically checking the scaling of the pseudo energies in the next section. As for the third type, the power series corrections are analyzed by extending the analysis in [43]. We explicitly solve the linearized equations including the leading term in the power series corrections in subsection 3.3. The free energy with the first $\mathcal{O}\left(L^{-2}\right)$ corrections is given in subsection 3.4. In subsection 3.5, the leading terms are re-expanded in terms of the parameters in the TBA equations, i.e. $M / A$. These results are applied to the amplitudes in section 5. The analysis of the corrections assures that the linearization gives a controlled approximation which is valid up to the relative corrections which are exponentially small in $A$ or of order $1 / A^{2}$.

\subsection{Linearization}

We consider the limit,

$$
A=a L, \quad M=m L, \quad a, m=\mathcal{O}(1), \quad L \gg 1 .
$$


The TBA equations (2.12) imply the following representation of $\varepsilon(\theta)$ and $\tilde{\varepsilon}(\theta)$,

$$
\varepsilon(\theta)=L(-a+m \cosh \theta)+r_{1}(\theta), \quad \tilde{\varepsilon}(\theta)=L \sqrt{2} m \cosh \theta+r_{2}(\theta),
$$

where $r_{i}(\theta)$ are positive numbers. Hence some terms in (2.12) are exponentially small in $L$ for $\theta \in \mathbb{R}$ :

$$
\begin{aligned}
\log \left(1+\mu^{-2} \mathrm{e}^{-\varepsilon}\right) & \sim \mathrm{e}^{-L\left(a+m \cosh \theta+r_{1}\right)}<\mathrm{e}^{-L(a+m \cosh \theta)} \\
\log \left(1+\mathrm{e}^{-\tilde{\varepsilon}}\right) & \sim \mathrm{e}^{-L\left(\sqrt{2} m \cosh \theta+r_{2}\right)}<\mathrm{e}^{-L \sqrt{2} m \cosh \theta} .
\end{aligned}
$$

By neglecting these terms, we approximate the first equation in (2.12) by the form containing only $\varepsilon$,

$$
\varepsilon(\theta)=-A+M \cosh \theta+K_{1} * \log \left(1+\mathrm{e}^{-\varepsilon}\right) .
$$

The other function $\tilde{\varepsilon}$ is then evaluated by using $\varepsilon$ as

$$
\tilde{\varepsilon}(\theta)=\sqrt{2} M \cosh \theta+K_{2} * \log \left(1+\mathrm{e}^{-\varepsilon}\right) .
$$

We assume that there are unique Fermi points $\theta= \pm B$ satisfying

$$
\varepsilon( \pm B)=0 .
$$

The explicit expression of $B$ in terms of $A$ and $M$ will be determined a posteriori. The convolution term is then divided into three pieces,

$$
K_{1} * \log \left(1+\mathrm{e}^{-\varepsilon}\right)=-\int_{-B}^{B} K_{1}\left(\theta-\theta^{\prime}\right) \varepsilon\left(\theta^{\prime}\right) \frac{d \theta^{\prime}}{2 \pi}+\Delta_{1}(\theta)+\Delta_{2}(\theta),
$$

where

$$
\begin{aligned}
& \Delta_{1}(\theta)=\left(\int_{\left|\theta^{\prime}-B\right|<\eta}+\int_{\left|\theta^{\prime}+B\right|<\eta}\right) K_{1}\left(\theta-\theta^{\prime}\right) \log \left(1+\mathrm{e}^{-\left|\varepsilon\left(\theta^{\prime}\right)\right|}\right) \frac{d \theta^{\prime}}{2 \pi}, \\
& \Delta_{2}(\theta)=\left(\int_{\theta^{\prime}>B+\eta}+\int_{\theta^{\prime}<-B-\eta}+\int_{\left|\theta^{\prime}\right|<B-\eta}\right) K_{1}\left(\theta-\theta^{\prime}\right) \log \left(1+\mathrm{e}^{-\left|\varepsilon\left(\theta^{\prime}\right)\right|}\right) \frac{d \theta^{\prime}}{2 \pi},
\end{aligned}
$$

and $\eta=O(1)$. By keeping only the first term in the right hand side of (3.4), one obtains a linearized TBA equation from (3.3),

$$
\varepsilon(\theta)=-A+M \cosh \theta-\int_{-B}^{B} K_{1}\left(\theta-\theta^{\prime}\right) \varepsilon\left(\theta^{\prime}\right) \frac{d \theta^{\prime}}{2 \pi} .
$$

Similarly we have

$$
\tilde{\varepsilon}(\theta)=\sqrt{2} M \cosh \theta-\int_{-B}^{B} K_{2}\left(\theta-\theta^{\prime}\right) \varepsilon\left(\theta^{\prime}\right) \frac{d \theta^{\prime}}{2 \pi} .
$$

The eq. (3.6) is identical to the $p=\frac{1}{2}$ case of (3.3) in [43] by

$$
\tilde{K}(\theta)=\delta(\theta)+\frac{K_{1}(\theta)}{2 \pi}, \quad \epsilon_{\text {Zamolodchikov }}(\theta)=-\varepsilon(\theta) .
$$

We can thus follow [43] to treat it analytically. 
In the limit (3.1), we expect from the linearized equations (3.6), (3.7) that

$$
\varepsilon(\theta) \sim L \epsilon(\theta), \quad \tilde{\varepsilon}(\theta) \sim L \tilde{\epsilon}(\theta) \quad \epsilon, \tilde{\epsilon} \sim O(1) .
$$

Under the assumption of this scaling, $\Delta_{2}$ is exponentially small in $L$. The scaling will be confirmed numerically in the next section. Below we are interested in the corrections to the linearized equations due to $\Delta_{1}(\theta)$. The Sommerfeld type argument results in, as the simplest approximation [42],

$$
\Delta_{1}(\theta) \sim \frac{\pi}{12\left|\varepsilon^{\prime}(B)\right|}\left(K_{1}(\theta-B)+K_{1}(\theta+B)\right) .
$$

This turns out to yield an $\mathcal{O}\left(L^{-2}\right)$ correction to $\epsilon(\theta)$. One can systematically analyze the higher power terms in $L^{-2}$ in principle by incorporating higher order terms from $\Delta_{1}$. We, however, restrict ourselves to the leading correction below.

\subsection{Analysis of the linearized TBA}

We recall the results in [43] which are necessary in the following discussion. Let us introduce

$$
\epsilon_{B}(\theta)= \begin{cases}-\frac{\varepsilon(\theta)}{L}=-\epsilon(\theta) & |\theta|<B \\ 0 & |\theta| \geq B\end{cases}
$$

Then the linearized TBA equation (3.6) is equivalent to,

$$
D_{\epsilon}(\theta)=\int_{-\infty}^{\infty} \tilde{K}\left(\theta-\theta^{\prime}\right) \epsilon_{B}\left(\theta^{\prime}\right) .
$$

The function $D_{\epsilon}(\theta)$ in the left hand side is defined by

$$
D_{\epsilon}(\theta)=Y(\theta)+Y(-\theta), \quad Y(\theta)= \begin{cases}X(\theta) & \theta>B \\ \frac{a-m \mathrm{e}^{\theta}}{2} & \theta<B .\end{cases}
$$

Here $X(\theta)$ is an unknown function of $\theta$.

The (inverse) Fourier transformation for an arbitrary smooth function $f(\theta)$ is defined by

$$
\widehat{f}(w)=\int_{-\infty}^{\infty} f(\theta) \mathrm{e}^{i w \theta} d \theta, \quad f(\theta)=\int_{-\infty}^{\infty} \widehat{f}(w) \mathrm{e}^{-i w \theta} \frac{d w}{2 \pi} .
$$

The kernel $\tilde{K}$ admits the factorization in the Fourier space,

$$
\widehat{K}(w)=\frac{1}{\widehat{K}_{+}(\omega) \widehat{K}_{-}(\omega)}, \quad \widehat{K}_{-}(w)=\widehat{K}_{+}(-w),
$$

where $\widehat{K}_{+}\left(\widehat{K}_{-}\right)$is analytic in the lower (upper) half plane and $\widehat{K}_{ \pm}(w) \rightarrow 1$ as $|w| \rightarrow \infty$. The explicit form of $\widehat{K}_{+}(w)$ reads,

$$
\widehat{K}_{+}(w)=\sqrt{\frac{2 \pi}{3}} e^{i \Delta w} \frac{\Gamma\left(1+\frac{3 w}{4} i\right)}{\Gamma\left(1+\frac{w}{4} i\right) \Gamma\left(\frac{1}{2}+\frac{w}{2} i\right)}, \quad \Delta=-\left(\frac{3}{4} \log \frac{3}{2}+\frac{1}{4} \log 2\right) .
$$


We further introduce $v(w)$ by

$$
v(w)=\mathrm{e}^{-i w B} \widehat{K}_{-}(w) Y(w) .
$$

Based on the Wiener-Hopf type factorization, it is shown in [43] that eq. (3.10) is casted into the integral equation for $v(w)$,

$$
\begin{aligned}
v(w) & =D_{v}(w)+\int_{-\infty}^{\infty} \frac{\mathrm{e}^{2 i w^{\prime} B}}{w+w^{\prime}+i 0^{+}} \alpha\left(w^{\prime}\right) v\left(w^{\prime}\right) \frac{d w^{\prime}}{2 \pi i}, \\
D_{v}(w) & =-i \frac{a \widehat{K}_{+}(0)}{2\left(w-i 0^{+}\right)}+i \frac{m e^{B} \widehat{K}_{+}(-i)}{2(w-i)} \\
\alpha(w) & =\frac{\widehat{K}_{+}(w)}{\widehat{K}_{-}(w)}
\end{aligned}
$$

The integral equation (3.11) concludes that $v(w)$ is an "almost" pole free function in the upper half plane and takes the form,

$$
\begin{aligned}
v(w) & =-i \frac{a \widehat{K}_{+}(0)}{2}\left(\frac{1}{w-i 0^{+}}+\frac{1}{w+i 0^{+}}\right)+i \frac{m e^{B} \widehat{K}_{+}(-i)}{2(w-i)}+\sum_{n \geq 1} \frac{\mathrm{e}^{-\frac{8}{3} n B}}{w+\frac{4}{3} n i} \alpha_{n} v_{n}, \\
\alpha_{n} & =\operatorname{res}_{w=\frac{4}{3} n i} \alpha(w), \quad v_{n}=v\left(\frac{4}{3} n i\right) .
\end{aligned}
$$

This determines the coefficients $v_{n}$ in a self-consistent way. In order to determine them, we utilize the condition $\varepsilon(B)=0$. This implies $v(w) \sim O\left(1 / w^{2}\right)$ for $|w| \gg 1$, which is written as

$$
i a \widehat{K}_{+}(0)-\frac{i m \mathrm{e}^{B}}{2} \widehat{K}_{+}(-i)=\int_{C_{+}} \mathrm{e}^{2 i w B} \alpha\left(w^{\prime}\right) v\left(w^{\prime}\right) \frac{d w^{\prime}}{2 \pi i} .
$$

Here the integration contour $C_{+}$encircles the positive imaginary axis of $w^{\prime}$ but it excludes the pole of $v\left(w^{\prime}\right)$ at $w^{\prime}=i 0^{+}$.

Set

$$
u(w)=-\frac{1+i w}{a \widehat{K}_{+}(0)} v(w)
$$

By using the condition (3.13), one rewrites the integral equation (3.11) in terms of $u(w)$ as

$$
\begin{aligned}
& u(w)=\frac{i}{w}+\int_{C_{+}} \frac{\mathrm{e}^{2 i w^{\prime} B}}{w+w^{\prime}+i 0^{+}} \rho\left(w^{\prime}\right) u\left(w^{\prime}\right) \frac{d w^{\prime}}{2 \pi i}, \\
& \rho(w)=\frac{1-i w}{1+i w} \alpha(w) .
\end{aligned}
$$

Here we understand that the pole at $w^{\prime}=-w$ in the integrand is outside of $C_{+}$.

Then one derives from eq. (3.14),

$$
\begin{aligned}
w_{n} & =\frac{1}{n}-\sum_{\ell=1}^{\infty} \frac{q^{\ell}}{\ell+n} b_{\ell} w_{\ell}, \quad n \in \mathbb{N}, \\
w_{n} & =\frac{4}{3} u\left(\frac{4}{3} n i\right) \\
b_{n} & =\frac{(-1)^{n}}{n !(n-1) !} \frac{\Gamma\left(\frac{n}{3}\right) \Gamma\left(\frac{3}{2}+\frac{2 n}{3}\right)}{\Gamma\left(-\frac{n}{3}\right) \Gamma\left(\frac{3}{2}-\frac{2 n}{3}\right)},
\end{aligned}
$$


where

$$
q=\mathrm{e}^{-8(B+\triangle) / 3} .
$$

Although the solution to eq. (3.15) is given by an infinite power series in $q$, it converges quickly for a large $a / m$.

The quantity of our interest is the pseudo energy, which is given by

$$
\widehat{\epsilon}_{B}(w)=\mathrm{e}^{i w B} \widehat{K}_{+}(w) v(w)+\mathrm{e}^{-i w B} \widehat{K}_{-}(w) v(-w),
$$

and its (inverse) Fourier transformation $\epsilon_{B}(\theta)$. In the inverse Fourier integral over $\theta$ with the region $-B<\theta<B$, we close the integration contour by adding the large semicircle in the upper half plane for the first term and in the lower half plane for the second. One immediately sees that the pole of $v(w)$ at $w=i$ is canceled by the zero of $\widehat{K}_{+}(w)$. The first term is thus evaluated by the contributions from the poles at $w=i 0^{+}, w=\frac{4}{3} n i(n>0, n \neq$ $0(\bmod 3))$. Similarly the second term is evaluated by those at $w=i 0^{-}, w=-\frac{4}{3} n i(n>$ $0, n \neq 0(\bmod 3))$. After simple manipulations, we obtain

$$
\epsilon(\theta)=-\epsilon_{B}(\theta)=-\frac{2 a}{3}-\sum_{\substack{n \geq 1 \\ n \neq 0(\bmod 3)}} c_{n} \cosh \frac{4 n}{3} \theta,
$$

for $|\theta|<B$, where we have used $\widehat{K}_{+}(0)=\sqrt{\frac{2}{3}}$. The coefficients $c_{n}$ are expressed by quantities already introduced in the above as

$$
\begin{aligned}
c_{n} & =2 i \mathrm{e}^{-\frac{4}{3} n B} v_{n} \operatorname{res}_{w=\frac{4}{3} n i} \widehat{K}_{+}, \\
\operatorname{res}_{w=\frac{4}{3} n i} \widehat{K}_{+} & =(-1)^{n} \frac{4 i}{3} \sqrt{\frac{2 \pi}{3}} \frac{\mathrm{e}^{-\frac{4 n}{3} \Delta}}{\Gamma(n) \Gamma\left(1-\frac{n}{3}\right) \Gamma\left(\frac{1}{2}-\frac{2 n}{3}\right)} .
\end{aligned}
$$

The other TBA function, $\tilde{\epsilon}=L^{-1} \tilde{\varepsilon}$, is also evaluated within the present linear approximation, which is given by

$$
\begin{aligned}
\tilde{\epsilon}(\theta) & =\sqrt{2} m \cosh \theta-\int_{-\infty}^{\infty} K_{2}\left(\theta-\theta^{\prime}\right) \epsilon\left(\theta^{\prime}\right) \frac{d \theta^{\prime}}{2 \pi} \\
& =\sqrt{2} m \cosh \theta+\int_{-\infty}^{\infty} \frac{\widehat{K}_{2}(w)}{2 \pi} \widehat{\epsilon}_{B}(w) \mathrm{e}^{-i w \theta} \frac{d w}{2 \pi} \\
& =\sqrt{2} m \cosh \theta+\int_{-\infty}^{\infty} \frac{\widehat{K}_{2}(w) \widehat{K}_{+}(w)}{2 \pi} v(w)\left(\mathrm{e}^{i w(B-\theta)}+\mathrm{e}^{i w(B+\theta)}\right) \frac{d w}{2 \pi} \\
\frac{\widehat{K}_{2}(w)}{2 \pi} & =\frac{\cosh \frac{\pi}{4} w}{\cosh \frac{\pi}{2} w} .
\end{aligned}
$$

Now we deform the integration contour by adding a semi-circle in the upper half plane. One easily verifies that $\widehat{K}_{2}(w) \widehat{K}_{+}(w)$ is analytic at $w=(2 n+1) i$. It is also easy to see that the pole contribution at $w=i$ (of $v(w)$ ) cancels $\sqrt{2} m \cosh \theta$. Thus, by calculating other pole contributions at $w=i 0^{+}($of $v(w))$ and at $w=4 / 3 n i(n \neq 0 \bmod 3)\left(\right.$ of $\left.\widehat{K}_{+}(w)\right)$, we have

$$
\tilde{\epsilon}(\theta)=\frac{2 a}{3}+\sum_{\substack{n \geq 1 \\ n \neq 0(\bmod 3)}}(-1)^{n} c_{n} \cosh \frac{4 n}{3} \theta .
$$


Finally we need to represent $B$ in terms of $a$ and $m$. This is achieved by taking account of the pole contributions in the right hand side of (3.13). The result is

$$
1-\sum_{n=1}^{\infty} \frac{b_{n}}{\frac{4}{3} n+1} q^{n} w_{n}=\left(\frac{y}{q}\right)^{\frac{3}{8}}, \quad y=\left(\frac{3 \sqrt{\pi} m}{2 a} \frac{\Gamma\left(\frac{3}{4}\right)}{\Gamma\left(\frac{1}{4}\right)}\right)^{\frac{8}{3}} .
$$

This equation fixes $q$ (and thus $B$ ) in terms of $y$. It also implies $B=\mathcal{O}\left(L^{0}\right)$.

In appendix A, we evaluate $\tilde{\epsilon}$ by directly plugging the expansion of $\epsilon$ (3.18) into (3.7). From this, one can see explicitly how the fractional powers of $e^{\theta}$ appear from the summation over its integral powers, in accordance with the periodicity required from the Y-system.

\section{$3.3 \mathcal{O}\left(L^{-2}\right)$ corrections}

One can extend the analysis by incorporating the $\mathcal{O}\left(L^{-2 k}\right)$ corrections to $\epsilon$. Here, we consider the $\mathcal{O}\left(L^{-2}\right)$ correction explicitly. The results at the leading order in this subsection reduce to those in the previous subsection.

By substituting eq. (3.9) into the right hand side of (3.4) and by neglecting $\Delta_{2}(\theta)$, one obtains an equation of the form (3.10). Then $Y(\theta)$ is modified by a term,

$$
Y(\theta)= \begin{cases}X(\theta) & \theta>B \\ \frac{a-m \mathrm{e}^{\theta}}{2}+\delta_{1}(\theta) & \theta<B,\end{cases}
$$

where

$$
\delta_{1}(\theta)=-\frac{\xi}{\cosh (\theta-B)}, \quad \xi=\frac{\pi}{12 L^{2}\left|\epsilon^{\prime}(B)\right|} .
$$

We argue in appendix $\mathrm{B}$ that $\epsilon^{\prime}(B)$ is of $\mathcal{O}\left(L^{0}\right)$. Thus $\delta_{1}$ represents the correction of $\mathcal{O}\left(L^{-2}\right)$ to the linearized TBA equation. We quantitatively estimate the consequence of this modification.

Note that

$$
\widehat{\delta}_{1}(w)=-\pi \xi \frac{\mathrm{e}^{i w B}}{\cosh \frac{\pi}{2} w}=2 i \xi \mathrm{e}^{i w B} \sum_{n=0}^{\infty}(-1)^{n}\left(\frac{1}{w-(2 n+1) i}-\frac{1}{w+(2 n+1) i}\right) .
$$

By using this, one can show that $v(w)$ satisfies eq. (3.11) with

$$
\begin{aligned}
& D_{v}(w)=-i \frac{a \widehat{K}_{+}(0)}{2\left(w-i 0^{+}\right)}+i \frac{m e^{B} \widehat{K}_{+}(-i)}{2(w-i)}+\delta_{-}(w) \mathrm{e}^{-i w B} \\
& \delta_{-}(w)=2 i \xi \mathrm{e}^{i w B} \sum_{n=0}^{\infty}(-1)^{n} \frac{\widehat{K}_{-}((2 n+1) i)}{w-(2 n+1) i} .
\end{aligned}
$$

The last term is the only modification due to the leading term from $\Delta_{1}(\theta)$. It however changes the analytic property of $v(w)$. As shown previously, $v(w)$ is almost pole free in the upper half plane of $w$ without $\delta_{-}(w)$. This time it has infinitely many poles in both half 
planes. More explicitly,

$$
\begin{aligned}
v(w)= & -i \frac{a \widehat{K}_{+}(0)}{2}\left(\frac{1}{w-i 0^{+}}+\frac{1}{w+i 0^{+}}\right)+i \frac{m e^{B} \widehat{K}_{+}(-i)}{2(w-i)} \\
& +\sum_{n \geq 1} \frac{\mathrm{e}^{-\frac{8}{3} n B}}{w+\frac{4}{3} n i} \alpha_{n} v\left(\frac{4}{3} n i\right)+\delta_{-}(w) \mathrm{e}^{-i w B}
\end{aligned}
$$

The convolution integral in (3.11) does not produce further terms as $\alpha(w) \delta_{-}(w)$ is regular at $w=(2 n+1) i, n \in \mathbb{Z}_{\geq 0}$.

The analogue of (3.13) reads,

$$
\begin{aligned}
-1+\frac{m \mathrm{e}^{B} \widehat{K}_{-}(i)}{2 a \widehat{K}_{+}(0)}+\frac{\xi_{1}}{L^{2}} \zeta_{c} & =\int_{C_{+}} \frac{i \mathrm{e}^{2 i w^{\prime} B}}{a \widehat{K}_{+}(0)} \alpha\left(w^{\prime}\right) v\left(w^{\prime}\right) \frac{d w^{\prime}}{2 \pi i} \\
\xi_{1}=\frac{2 \xi L^{2}}{a \widehat{K}_{+}(0)}, \quad \zeta_{c} & =\frac{1}{2}+\sum_{\ell=0}^{\infty}(-1)^{\ell}\left(\widehat{K}_{-}((2 \ell+1) i)-1\right)
\end{aligned}
$$

Here $\xi_{1}=\mathcal{O}\left(L^{0}\right)$, and it is estimated in appendix B. By utilizing this, one derives the equation for $u(w)$,

$$
u(w)=\frac{i}{w}+\frac{\xi_{1}}{L^{2}} \sum_{n=1}^{\infty} \frac{2 n(-1)^{n} i}{w-(2 n+1) i} \widehat{K}_{-}((2 n+1) i)+\int_{C_{+}} \frac{\mathrm{e}^{2 i w^{\prime} B}}{w+w^{\prime}+i 0^{+}} \rho\left(w^{\prime}\right) u\left(w^{\prime}\right) \frac{d w^{\prime}}{2 \pi i} .
$$

As mentioned previously, the pole at $w^{\prime}=-w$ in the integrand is outside of $C_{+}$. From this, we again obtain a set of algebraic equations for $w_{n}$,

$$
w_{n}=\frac{1}{n}+\frac{4 \xi_{1}}{3 L^{2}} \sum_{\ell=1}^{\infty}(-1)^{\ell} \frac{2 \ell}{\frac{4 n}{3}-(2 \ell+1)} \widehat{K}_{-}((2 \ell+1) i)-\sum_{\ell \geq 1} \frac{q^{\ell}}{n+\ell} b_{\ell} w_{\ell},
$$

where $q$ is defined in (3.16). For a given sequence $\omega_{n, 0}(n>0)$ of $\mathcal{O}(1)$, let $\omega_{n}$ be a solution to

$$
\omega_{n}=\omega_{n, 0}-\sum_{\ell \geq 1} \frac{q^{\ell}}{n+\ell} b_{\ell} \omega_{\ell}
$$

It has a power series solution of the form,

$$
\omega_{n}=\sum_{\ell=0}^{\infty} \omega_{n, \ell} q^{\ell}
$$

The coefficients $\omega_{n, \ell}(\ell \geq 1)$ are uniquely determined by $\omega_{n, 0}$. The first few coefficients read explicitly

$$
\begin{aligned}
& \omega_{n, 1}=-\frac{1}{n+1} b_{1} \omega_{1,0}, \quad \omega_{n, 2}=\frac{1}{2 n+2} b_{1}^{2} \omega_{1,0}-\frac{1}{n+2} b_{2} \omega_{2,0} \\
& \omega_{n, 3}=-\frac{1}{4(n+1)} b_{1}^{3} \omega_{1,0}+\frac{1}{3(n+2)} b_{1} b_{2} \omega_{1,0}+\frac{1}{3(n+1)} b_{1} b_{2} \omega_{2,0}-\frac{1}{n+3} b_{3} \omega_{3,0} .
\end{aligned}
$$


The solution to (3.24) is thus written in the form,

$$
w_{n}=w_{n}^{(0)}(q)+\frac{w_{n}^{(1)}(q)}{L^{2}}, \quad w_{n}^{(0)}(q)=\sum_{\ell=0}^{\infty} \gamma_{n \ell} q^{\ell}, \quad w_{n}^{(1)}(q)=\sum_{\ell=0}^{\infty} \kappa_{n \ell} q^{\ell} .
$$

In the above we have used

$$
\gamma_{n \ell}=\left.\omega_{n, \ell}\right|_{\omega_{n, 0} \rightarrow 1 / n}, \quad \kappa_{n \ell}=\left.\omega_{n, \ell}\right|_{\omega_{n, 0} \rightarrow \kappa_{n 0}}
$$

and

$$
\kappa_{n 0}=\frac{4 \xi_{1}}{3} \sum_{\ell=1}^{\infty}(-1)^{\ell} \frac{2 \ell}{\frac{4 n}{3}-(2 \ell+1)} \widehat{K}_{-}((2 \ell+1) i) .
$$

Note that $w_{n}^{(0)}(q)$ and $w_{n}^{(1)}(q)$ are not decompositions of $w_{n}$ into $\mathcal{O}\left(L^{0}\right)$ and $\mathcal{O}\left(L^{-2}\right)$ terms. As will be shown below, $q$ is given by a power series in $L^{-2}$.

Next we represent $q$ in terms of $y$ defined in (3.20). This is achieved by rewriting (3.22) in terms of $w_{n}$,

$$
1-\sum_{\ell=1}^{\infty} \frac{b_{\ell}}{\frac{4}{3} \ell+1} q^{\ell} w_{\ell}=\left(1+\frac{\nu}{L^{2}}\right)\left(\frac{y}{q}\right)^{\frac{3}{8}} .
$$

Here we introduced

$$
\nu=\frac{2 \xi_{1} \mathrm{e}^{-B} \widehat{K}_{+}(0) a}{\widehat{K}_{-}(i) m} \zeta_{c}
$$

which is again of $\mathcal{O}\left(L^{0}\right)$.

In order to solve this we set

$$
q=q^{(0)}+q^{(1)} L^{-2}+\mathcal{O}\left(L^{-4}\right) .
$$

By substituting (3.26) and (3.30) into (3.29) one obtains equations of $\mathcal{O}\left(L^{0}\right), \mathcal{O}\left(L^{-2}\right)$ as

$$
\begin{gathered}
1-\sum_{\ell=1}^{\infty} \frac{b_{\ell}\left(q^{(0)}\right)^{\ell} \widetilde{w}_{\ell}^{(0)}}{\frac{4}{3} \ell+1}=\left(\frac{y}{q^{(0)}}\right)^{\frac{3}{8}}, \\
-q^{(1)} \sum_{\ell=1}^{\infty} \frac{\ell b_{\ell}\left(q^{(0)}\right)^{\ell-1} \widetilde{w}_{\ell}^{(0)}}{\frac{4}{3} \ell+1}-\sum_{\ell=1}^{\infty} \frac{b_{\ell}\left(q^{(0)}\right)^{\ell} \widetilde{w}_{\ell}^{(1)}}{\frac{4}{3} \ell+1}=\left(\frac{y}{q^{(0)}}\right)^{\frac{3}{8}}\left(\nu-\frac{3 q^{(1)}}{8 q^{(0)}}\right),
\end{gathered}
$$

respectively. The coefficients $\widetilde{w}_{\ell}^{(0)}$ and $\widetilde{w}_{\ell}^{(1)}$ are given by

$$
\widetilde{w}_{n}^{(0)}=\sum_{\ell=0}^{\infty} \gamma_{n \ell}\left(q^{(0)}\right)^{\ell}, \quad \widetilde{w}_{n}^{(1)}=q^{(1)} \sum_{\ell=0}^{\infty} \ell \gamma_{n \ell}\left(q^{(0)}\right)^{\ell-1}+\sum_{\ell=0}^{\infty} \kappa_{n \ell}\left(q^{(0)}\right)^{\ell} .
$$

Note that they are now decompositions of $w_{\ell}$ into $\mathcal{O}\left(L^{0}\right)$ and $\mathcal{O}\left(L^{-2}\right)$ terms,

$$
w_{\ell}=\widetilde{w}_{\ell}^{(0)}+L^{-2} \widetilde{w}_{\ell}^{(1)}+\mathcal{O}\left(L^{-4}\right) .
$$

We assume expansions

$$
q^{(0)}=y+\sum_{\ell=2}^{\infty} q_{\ell}^{(0)} y^{\ell}, \quad q^{(1)}=\frac{8}{3} \nu y+\sum_{\ell=2}^{\infty} q_{\ell}^{(1)} y^{\ell},
$$


and substitute them into (3.31). Then the coefficients $q_{\ell}^{(0)}$ and $q_{\ell}^{(1)}$ are determined order by order in $y$. For later use, we list $q_{\ell}^{(0)}$ for $\ell=2,3,4$ :

$$
q_{2}^{(0)}=\frac{8}{7} b_{1}, \quad q_{3}^{(0)}=\frac{80}{49} b_{1}^{2}+\frac{4}{11} b_{2}, \quad q_{4}^{(0)}=\frac{382}{147} b_{1}^{3}+\frac{320}{231} b_{1} b_{2}+\frac{8}{45} b_{3} .
$$

The key quantity $\widehat{\epsilon}_{B}(w)$ has formally the same form as (3.17). We take its inverse Fourier transformation. The extra poles of $v(w)$ at $w=(2 n+1) i(n \geq 0)$ do not play a role as the combination $\widehat{K}_{+}(w) v(w)$ is regular at these points. The integration contour is closed in the upper (lower) half plane for the first (second) term. We then arrive at

$$
\epsilon(\theta)=-\frac{2 a}{3}-\sum_{\substack{n \geq 1 \\ n \neq 0(\bmod 3)}} c_{n} \cosh \frac{4 n}{3} \theta, \quad c_{n}=2 i \mathrm{e}^{-\frac{4}{3} n B} v_{n} \mathrm{res}_{w=\frac{4}{3} n i} \widehat{K}_{+}(w) .
$$

This is formally identical to the one without $\Delta_{1}(\theta)$. The coefficients $v_{n}$, however, contain higher order terms of $\mathcal{O}\left(L^{-2 k}\right)$ and the coefficients $c_{n}$ also contain them similarly. The $\mathcal{O}\left(L^{0}\right)$ and $\mathcal{O}\left(L^{-2}\right)$ terms are given explicitly in terms of other coefficients obtained so far,

$$
\begin{aligned}
c_{n} & =\left(c_{n}^{(0)}+\frac{c_{n}^{(1)}}{L^{2}}\right) a+\mathcal{O}\left(\frac{1}{L^{4}}\right), \\
c_{n}^{(0)} & =\left(q^{(0)}\right)^{\frac{n}{2}} \widetilde{w}_{n}^{(0)} \widetilde{c}_{n} \frac{3 \widehat{K}_{+}(0)}{4\left(\frac{4}{3} n-1\right)}, \quad c_{n}^{(1)}=\left(q^{(0)}\right)^{\frac{n}{2}}\left(\widetilde{w}_{n}^{(1)}+\frac{n q^{(1)}}{2 q^{(0)}} \widetilde{w}_{n}^{(0)}\right) \widetilde{c}_{n} \frac{3 \widehat{K}_{+}(0)}{4\left(\frac{4}{3} n-1\right)}, \\
\widetilde{c}_{n} & =\frac{8}{3} \sqrt{\frac{2 \pi}{3}} \frac{(-1)^{n+1}}{\Gamma(n) \Gamma\left(1-\frac{n}{3}\right) \Gamma\left(\frac{1}{2}-\frac{2 n}{3}\right)} .
\end{aligned}
$$

By using these, we have

$$
\frac{\epsilon(\theta)}{a}=-\frac{2}{3}-\sum_{\substack{n \geq 1 \\ n \neq 0(\bmod 3)}} c_{n}^{(0)} \cosh \frac{4 n \theta}{3}-\frac{1}{L^{2}} \sum_{\substack{n \geq 1 \\ n \neq 0(\bmod 3)}} c_{n}^{(1)} \cosh \frac{4 n \theta}{3}+\mathcal{O}\left(\frac{1}{L^{4}}\right) .
$$

We can similarly estimate the correction to $\tilde{\epsilon}(\theta)$,

$$
\tilde{\epsilon}(\theta)=\sqrt{2} m \cosh \theta-\int_{-\infty}^{\infty} K_{2}\left(\theta-\theta^{\prime}\right) \epsilon\left(\theta^{\prime}\right) \frac{d \theta^{\prime}}{2 \pi}+\xi\left(K_{2}(\theta-B)+K_{2}(\theta+B)\right) .
$$

The convolution term is again given by

$$
\int_{-\infty}^{\infty} \frac{\widehat{K}_{2}(w) \widehat{K}_{+}(w)}{2 \pi} v(w)\left(\mathrm{e}^{i w(B-\theta)}+\mathrm{e}^{i w(B+\theta)}\right) \frac{d w}{2 \pi} .
$$

As $|\theta|<B$ we close the contour in the upper half plane. There are contributions from the poles at $w=i 0^{+}, w=i$ and $w=\frac{4}{3} n i(n \neq 0(\bmod 3))$ as before. In addition, there appear additional contributions at $w=(2 n+1) i$, originated from the last term in (3.21). After a simple calculation we find that the additional term cancels the last two terms in (3.36),

$$
\xi\left(K_{2}(\theta-B)+K_{2}(\theta+B)\right)-\sum_{n=0} 8 \xi(-1)^{n} \sin \frac{2 n+3}{4} \mathrm{e}^{-(2 n+1) B} \cosh (2 n+1) \theta=0 .
$$


Thus we have

$$
\frac{\tilde{\epsilon}(\theta)}{a}=\frac{2}{3}+\sum_{\substack{n \geq 1 \\ n \neq 0(\bmod 3)}}(-1)^{n} c_{n}^{(0)} \cosh \frac{4 n \theta}{3}+\frac{1}{L^{2}} \sum_{\substack{n \geq 1 \\ n \neq 0(\bmod 3)}}(-1)^{n} c_{n}^{(1)} \cosh \frac{4 n \theta}{3}+\mathcal{O}\left(\frac{1}{L^{4}}\right)
$$

This and (3.35) are consistent with the periodicity property of the Y-system (2.15).

\subsection{Free energy}

The free energy $A_{\text {free }}$ is represented in terms of the TBA functions as in (2.16). As before, we drop the terms which are exponentially small by assuming the scaling (3.8), and write

$$
A_{\text {free }}=-\Delta \mathcal{E}+\Delta_{F}
$$

where

$$
\begin{aligned}
\Delta \mathcal{E} & =\int_{-B}^{B} M \cosh \theta \varepsilon(\theta) \frac{d \theta}{2 \pi}=-\int_{-\infty}^{\infty} m L^{2} \cosh \theta \epsilon_{B}(\theta) \frac{d \theta}{2 \pi}, \\
\Delta_{F} & =\left(\int_{|B-\theta|<\eta}+\int_{|B+\theta|<\eta}\right) m L \cosh \theta \log \left(1+\mathrm{e}^{-|\varepsilon(\theta)|}\right) \frac{d \theta}{2 \pi} .
\end{aligned}
$$

For the moment, we concentrate on $\Delta \mathcal{E}$. One can show that the following relation, derived in [43], is still valid even in the presence of $\Delta_{1}(\theta)$,

$$
\Delta \mathcal{E}=-\frac{m L^{2}}{2 \pi} \widehat{\epsilon}_{B}(-i)
$$

Thanks to the integral equation for $\widehat{\epsilon}_{B}$, we have

$$
\Delta \mathcal{E}=-\frac{m L^{2} \mathrm{e}^{B} \widehat{K}_{-}(i)}{2 \pi}\left(D_{v}(-i)+\int_{C_{+}^{\prime}} \frac{\mathrm{e}^{2 i w B}}{w-i} \alpha(w) v(w) \frac{d w}{2 \pi i}\right) .
$$

Here the contour $C_{+}^{\prime}$ encircles the positive imaginary axis, which includes $w=i$. We use the explicit form of $D_{v}(w)$ and the "boundary condition" (3.22) to represent this as

$$
\begin{aligned}
\Delta \mathcal{E}= & -\frac{m a L^{2} \mathrm{e}^{B} \widehat{K}_{-}(i) \widehat{K}_{+}(0)}{4 \pi} \\
& \times\left(1-\int_{C_{+}} \frac{d w}{2 \pi i} \mathrm{e}^{2 i w B} \frac{\rho(w) u(w)}{w-i}+\frac{\xi_{1}}{L^{2}} \sum_{n=0}^{\infty}(-1)^{n} \frac{n}{n+1} \widehat{K}_{-}((2 n+1) i)\right) .
\end{aligned}
$$

We write $\Delta \mathcal{E}=\Delta \mathcal{E}_{1}+\Delta \mathcal{E}_{2}$ where $\Delta \mathcal{E}_{1}$ stands for the contribution from the pole at $w=i$ in the integral. Explicitly,

$$
\frac{\Delta \mathcal{E}_{1}}{L^{2}}=\frac{m^{2}}{4}+\frac{a m}{2} \mathrm{e}^{-B} \widehat{K}_{+}(0) \frac{\xi_{1}}{L^{2}}
$$

Here we used

$$
u(i)=\frac{-i}{a \widehat{K}_{+}(0)} \operatorname{res}_{w=i} v(w)=\frac{\widehat{K}_{-}(i)}{a \widehat{K}_{+}(0)}\left(\frac{m \mathrm{e}^{B}}{2}+2 \xi\right)
$$


Similarly,

$$
\begin{aligned}
\frac{\Delta \mathcal{E}_{2}}{L^{2}}= & -\frac{\left(a \widehat{K}_{+}(0)\right)^{2}}{2 \pi}\left(u(i)-\frac{\xi_{1}}{L^{2}} \widehat{K}_{-}(i)\right) \\
& \times\left(1-\int_{C_{+}^{\prime} \backslash\{i\}} \frac{\mathrm{e}^{2 i w B}}{w-i} \rho(w) u(w) \frac{d w}{2 \pi i}+\frac{\xi_{1}}{L^{2}} \sum_{n=0}^{\infty}(-1)^{n} \frac{n}{n+1} \widehat{K}_{-}((2 n+1) i)\right) .
\end{aligned}
$$

The content of the first bracket in the right hand side is evaluated by use of (3.23),

$$
\begin{aligned}
u(i)-\frac{\xi_{1}}{L^{2}} \widehat{K}_{-}(i) & =1-\sum_{n=1} \frac{3}{4 n+3} q^{n} b_{n} w_{n}-\frac{\xi_{1}}{L^{2}} \zeta_{c}=\alpha^{(0)}+\frac{\alpha^{(1)}}{L^{2}}+\mathcal{O}\left(\frac{1}{L^{4}}\right) \\
\alpha^{(0)} & =1-\sum_{n=1}^{\infty} \frac{3}{4 n+3}\left(q^{(0)}\right)^{n} b_{n} \widetilde{w}_{n}^{(0)} \\
\alpha^{(1)} & =-\sum_{n=1}^{\infty}\left(\frac{3}{4 n+3}\left(q^{(0)}\right)^{n} b_{n} \widetilde{w}_{n}^{(1)}+\frac{3 n}{4 n+3}\left(q^{(0)}\right)^{n-1} q^{(1)} b_{n} \widetilde{w}_{n}^{(0)}\right)-\xi_{1} \zeta_{c} .
\end{aligned}
$$

The second bracket term is also decomposed into $\beta^{(0)}+\beta^{(1)} L^{-2}+\mathcal{O}\left(L^{-4}\right)$ where

$$
\begin{aligned}
& \beta^{(0)}=1+\sum_{n=1} \frac{3}{4 n-3}\left(q^{(0)}\right)^{n} b_{n} \widetilde{w}_{n}^{(0)} \\
& \beta^{(1)}=\sum_{n=1}\left(\frac{3}{4 n-3}\left(q^{(0)}\right)^{n} b_{n} \widetilde{w}_{n}^{(1)}+\frac{3 n}{4 n-3}\left(q^{(0)}\right)^{n-1} q^{(1)} b_{n} \widetilde{w}_{n}^{(0)}\right)+\xi_{1}\left(\zeta_{c}-\zeta_{c}^{\prime}\right),
\end{aligned}
$$

with

$$
\zeta_{c}^{\prime}=\log 2+\sum_{\ell=0}^{\infty} \frac{(-1)^{\ell}}{\ell+1}\left(\widehat{K}_{-}((2 \ell+1) i)-1\right)
$$

Thus we have

$$
\begin{aligned}
\frac{\Delta \mathcal{E}}{L^{2}} & =\Delta \mathcal{E}^{(0)}+\frac{\Delta \mathcal{E}^{(1)}}{L^{2}}+\mathcal{O}\left(\frac{1}{L^{4}}\right), \\
\Delta \mathcal{E}^{(0)} & =\frac{m^{2}}{4}-\frac{\left(a \widehat{K}_{+}(0)\right)^{2}}{2 \pi} \alpha^{(0)} \beta^{(0)}, \\
\Delta \mathcal{E}^{(1)} & =\frac{a m \xi_{1}}{2} \mathrm{e}^{-B} \widehat{K}_{+}(0)-\frac{\left(a \widehat{K}_{+}(0)\right)^{2}}{2 \pi}\left(\alpha^{(0)} \beta^{(1)}+\alpha^{(1)} \beta^{(0)}\right) .
\end{aligned}
$$

As for $\Delta_{F}$, the lowest order approximation gives

$$
\Delta_{F}=\frac{\pi}{12\left|\epsilon^{\prime}(B)\right|} 2 m \cosh B+\mathcal{O}\left(\frac{1}{L^{2}}\right)=\delta_{F}+\mathcal{O}\left(\frac{1}{L^{2}}\right)
$$

where

$$
\delta_{F}=\frac{\pi m}{12\left|\epsilon^{\prime}(B)\right|}\left(\left(q^{(0)}\right)^{\frac{3}{8}} e^{\Delta}+\left(q^{(0)}\right)^{-\frac{3}{8}} e^{-\Delta}\right) .
$$

We have substituted (3.16) and (3.30) into $e^{B}$. Thus, we obtain

$$
A_{\text {free }}=-L^{2} \Delta \mathcal{E}^{(0)}-\Delta \mathcal{E}^{(1)}+\delta_{F}+\mathcal{O}\left(\frac{1}{L^{2}}\right) .
$$




\subsection{Leading order expansions in $1 / L$}

In section 5, we will apply the results in this section for the 6-point MHV amplitudes at strong coupling. To express the expansions of $\epsilon, \tilde{\epsilon}$ and $A_{\text {free }}$, and hence the amplitudes in terms of the parameters in the TBA equations $M, A$ or $y$ in (3.20), we first solve (3.25) to find $\tilde{w}_{n}^{(0)}, \tilde{w}_{n}^{(1)}$ in (3.32). We then solve (3.31) to find $q^{(0)}, q^{(1)}$ in terms of $y$, and substitute them into (3.35), (3.37) and (3.43) with (3.34), (3.39), (3.40), (3.41) and (3.42).

Since we are interested in the expansions for large $L$, we will focus in section 5 on the expansions at the leading order in $1 / L^{2}$. In this case, one needs $\tilde{w}_{n}^{(0)}$ and $q^{(0)}$ only and does not need to take into account $c_{n}^{(1)}, \Delta \mathcal{E}^{(1)}$ and $\delta_{F}$. We then have simple expansions of $\epsilon, \tilde{\epsilon}$ :

$$
\begin{aligned}
& \epsilon(\theta)=a \sqrt{\pi} \cdot \sum_{n=0}^{\infty} e_{n}(\theta) y^{n / 2}+\mathcal{O}\left(L^{-2}\right), \\
& \tilde{\epsilon}(\theta)=a \sqrt{\pi} \cdot \sum_{n=0}^{\infty}(-1)^{n+1} e_{n}(\theta) y^{n / 2}+\mathcal{O}\left(L^{-2}\right),
\end{aligned}
$$

for $|\theta|<B$, where the coefficients $e_{n}$ for small $n$ are found to be

$$
\begin{array}{rlrl}
e_{0}(\theta) & =-\frac{2}{3 \sqrt{\pi}}, & e_{1}(\theta) & =-\frac{4 \cosh \frac{4}{3} \theta}{\Gamma\left(-\frac{1}{6}\right) \Gamma\left(\frac{2}{3}\right)}, \\
e_{2}(\theta) & =\frac{\frac{2}{5} \cosh \frac{8}{3} \theta}{\Gamma\left(-\frac{5}{6}\right) \Gamma\left(\frac{1}{3}\right)}, & e_{3}(\theta)=-\frac{\frac{2}{7} b_{1} \cosh \frac{4}{3} \theta}{\Gamma\left(-\frac{1}{6}\right) \Gamma\left(\frac{2}{3}\right)}, \\
e_{4}(\theta)=\frac{\frac{1}{78} \cosh \frac{16}{3} \theta}{\Gamma\left(-\frac{13}{6}\right) \Gamma\left(-\frac{1}{3}\right)}+\frac{\frac{4}{21} b_{1} \cosh \frac{8}{3} \theta}{\Gamma\left(-\frac{5}{6}\right) \Gamma\left(\frac{1}{3}\right)} & \\
e_{5}(\theta)=-\frac{\frac{1}{510} \cosh \frac{20}{3} \theta}{\Gamma\left(-\frac{17}{6}\right) \Gamma\left(-\frac{2}{3}\right)}-\frac{\left(\frac{9}{49} b_{1}^{2}+\frac{2}{33} b_{2}\right) \cosh \frac{4}{3} \theta}{\Gamma\left(-\frac{1}{6}\right) \Gamma\left(\frac{2}{3}\right)}, & \\
e_{6}(\theta)=\frac{2}{\Gamma\left(-\frac{13}{6}\right)} b_{1} \cosh \frac{16}{3} \theta \\
\Gamma\left(-\frac{1}{3}\right)
\end{array}
$$

with $b_{n}$ given in (3.15).

Similarly, the leading order term of the free energy is [43]

$$
f:=-A_{\text {free }} / L^{2}=\Delta \mathcal{E}^{(0)}+\mathcal{O}\left(L^{-2}\right), \quad \Delta \mathcal{E}^{(0)}=\frac{m^{2}}{4}-\frac{a^{2}}{\pi} \sum_{n=0}^{\infty} k_{n} y^{n},
$$

where we have introduced $f$ so that the leading terms are of $\mathcal{O}\left(L^{0}\right)$. The coefficients $k_{n}$ for small $n$ are $^{2}$

$$
\begin{array}{rlrl}
k_{0} & =\frac{1}{3}, & k_{1} & =\frac{6}{7} b_{1}, \\
k_{2}=\frac{6}{49} b_{1}^{2}+\frac{3}{55} b_{2}, & k_{3} & =\frac{57}{686} b_{1}^{3}+\frac{6}{77} b_{1} b_{2}+\frac{2}{135} b_{3}, \\
k_{4} & =\frac{29}{343} b_{1}^{4}+\frac{4}{33} b_{1}^{2} b_{2}+\frac{3}{242} b_{2}^{2}+\frac{4}{105} b_{1} b_{3}+\frac{3}{494} b_{4} .
\end{array}
$$

\footnotetext{
${ }^{2}$ We correct typos in $k_{3}, k_{4}$ in [43].
} 
The $\mathcal{O}\left(y^{0}\right)$ terms agree with the UV limits in (2.21). Though these expansions are based on the TBA equations of the form (2.12) which are valid for $|\operatorname{Im} \theta|<\pi / 4$, they are analytically continued for any $\operatorname{Im} \theta$.

Overlap with the small mass expansion: though we have discussed the case where both $A$ and $M$ are large, the results may be continued to small $M$, or those of the small $M$ expansions with other parameters fixed [38] may be continued to the regime of our expansions. Thus as a non-trivial check, they can be compared. Indeed, taking into account the relation of the notation here and that in [38],

$$
\log Y_{1, \text { there }}(\theta+i \varphi)=\varepsilon(\theta)+A, \quad \ell_{\text {there }}=M, \quad \phi_{\text {there }}=\frac{2}{3 i} A,
$$

we find that the expansions of $Y_{1, \text { there }}$ and $A_{\text {free }}$ in [38] precisely reproduce for large $A$ the leading terms of our expansions,

$$
a \sqrt{\pi} e_{1}(0) y^{1 / 2}, \quad-\frac{a^{2}}{\pi} k_{1} y
$$

\section{Numerical analysis}

In the previous section, the TBA equations for the 6-point amplitudes (2.12) for large $A$ and $M$ were shown to reduce to (3.4) up to exponentially small terms in $L \sim A$. The corrections from $\Delta_{1}$ are of $\mathcal{O}\left(L^{-1}\right)$ and hence relatively of $\mathcal{O}\left(L^{-2}\right)$, and those from $\Delta_{2}$ are again exponentially small from the assumption on the scaling of the pseudo energies (3.8). Once these corrections are dropped, the TBA equations further reduce to the linearized TBA equations (3.6) and (3.7). These are solved by the method in [43]. By extending the analysis in [43], the relatively $\mathcal{O}\left(L^{-2}\right)$ terms for $\varepsilon, \tilde{\varepsilon}$ were explicitly evaluated. Similarly, the free energy $A_{\text {free }}$ reduces to (3.38) up to terms which are exponentially small or expectedly exponentially small by the scaling (3.8). The leading contributions are of $\mathcal{O}\left(L^{2}\right)$, and the others are of $\mathcal{O}\left(L^{0}\right)$ and hence relatively of $\mathcal{O}\left(L^{-2}\right)$ again. By following and extending the analysis in [43], we evaluated both contributions explicitly.

\subsection{Dependence on $L$}

Now, we confirm the $L$-dependence of the expansions (3.35), (3.37) and (3.43).

This also serves as a check of our assumption on the scaling (3.8), which assures that $\Delta_{2}$ and a similar term dropped in (3.38) are indeed exponentially small in $L$. For this purpose, we first consider the pseudo energy at the special value of $\theta=0$. From the results in the previous section, we have

$$
\epsilon(0)=a_{1}+\frac{a_{2}}{L^{2}}+\mathcal{O}\left(L^{-4}\right),
$$

up to (expectedly) exponentially small terms with

$$
a_{1}=a \sqrt{\pi} \cdot \sum_{n=0}^{\infty} e_{n}(0) y^{n / 2}, \quad a_{2}=-a \sum_{\substack{n \geq 1 \\ n \neq 0(\bmod 3)}} c_{n}^{(1)} .
$$




\begin{tabular}{|l|l|l|l|l|}
\hline$m$ & $a_{1}$ & $a_{2}$ & $d_{1}$ & $d_{2}$ \\
\hline $10^{-1 / 4}$ & -0.35979817 & 0.34641 & -0.055404898 & -0.63726 \\
$10^{-2 / 4}$ & -0.52340676 & 0.15038 & -0.087142723 & -0.54386 \\
$10^{-3 / 4}$ & -0.5999064791 & 0.070976 & -0.09949568311 & -0.52780 \\
$10^{-1}$ & -0.63561331932 & 0.033492 & -0.103882817866 & -0.52450 \\
\hline
\end{tabular}

Table 1. Parameters for the $L$-dependence of $\epsilon(0)$ and $f$ from the results in section 3 .

One has a similar expression for $\tilde{\epsilon}(0)$. As for the free energy, the $L$-dependence reads

$$
f=d_{1}+\frac{d_{2}}{L^{2}}+\mathcal{O}\left(\frac{1}{L^{4}}\right)
$$

up to (expectedly) exponentially small terms, where $d_{1}=\Delta \mathcal{E}^{(0)}$ in (3.45) and $d_{2}=\Delta \mathcal{E}^{(1)}$ $\delta_{F}$ in (3.43).

Table 1 summarizes the numerical values of $a_{1}, a_{2}$ and $d_{1}, d_{2}$, e.g. for $a=1$ and $m=10^{-\ell / 4}(\ell=1, \ldots, 4)$. Here, terms up to order $y^{3} \sim(m / a)^{8}$ are included. To evaluate $\kappa_{n 0}$ in $(3.28), \widehat{K}_{-}((2 \ell+1) i)$ is rewritten as $\left(\widehat{K}_{-}((2 \ell+1) i)-1\right)+1$ and the remainder is separated as in $\zeta_{c}, \zeta_{c}^{\prime}$, so that the quantity in the parentheses is vanishing for large $\ell$. $\zeta_{c}, \zeta_{c}^{\prime}$ and $\kappa_{n 0}$ are estimated by including terms up to $\ell=10^{4}$ in the summation. As we have truncated the expansion, the correction is expected to be small for small $\mathrm{m}$.

We compare these with numerical results. To this end, we have solved the original TBA equations (2.12) numerically by iteration as described below (2.2). The free energy $f$ has also been evaluated numerically by (2.16). We set $a=1, m=10^{-\ell / 4}(\ell=1, \ldots, 4)$, and $L=L_{k}:=10^{\frac{1}{2}+\frac{k}{40}}(k=0, \ldots, 100)$. We also set the cut off of the integration over $\theta$ at $\theta= \pm \theta_{\max }$ with $\theta_{\max }=10 \cdot\left|\log \frac{2}{M}(A+308)\right|$. The interval $|\theta| \leq \theta_{\max }$ is discretized by $N=10^{16}$ points. The iteration stops when the change of the free energy (2.16) by one iteration becomes $2 \pi \cdot 10^{-16}$, or the number of the iterations reaches $n_{\max }=100$.

The numerical data $\epsilon_{\text {num }}(0)$ of $\epsilon(0)$ can be fitted by the function $\epsilon_{\text {fit }}(0)=a_{1}+a_{2} / L^{a_{3}}$. For the data, e.g. from $L=L_{k}$ with $k=71, \ldots, 100$ and $m=10^{-3 / 4}$, we obtain

$$
a_{1}=-0.5999064788, \quad a_{2}=0.07104736981, \quad a_{3}=2.000603100 .
$$

A similar fitting works also for $\tilde{\epsilon}(0)$. As for the free energy, the data $f_{\text {num }}$ of $f$ for the same $L_{k}$ and $m$ are fitted by $f_{\text {fit }}=d_{1}+d_{2} / L^{d_{3}}$ with

$$
d_{1}=-0.099495683121, \quad d_{2}=-0.5264772531, \quad d_{3}=2.000003585 .
$$

These are consistent with (4.1), (4.2) and the values of $\left(a_{1}, a_{2}\right)$ and $\left(d_{1}, d_{2}\right)$ in table 1.

Figure 2(a) and 2(b) are $\log -\log$ plots of $\epsilon(0)$ and $f$, respectively. In (a), the dots represent the numerical data, $\log \left(\epsilon_{\text {num }}(0)-a_{1}\right)$. The solid line represents the fitting function, $\log \left(\epsilon_{\mathrm{fit}}(0)-a_{1}\right)=\log \left(a_{2} / L^{a_{3}}\right)$. The coefficients $a_{i}$ are given in (4.3). In (b), the dots represent the numerical data, $\log \left(d_{1}-f_{\text {num }}(0)\right)$. The solid line represents the fitting function, $\log \left(d_{1}-f_{\text {fit }}\right)=\log \left(d_{2} / L^{d_{3}}\right)$. The coefficients $d_{i}$ are given in (4.4). The numerical data are well fitted for large $L$. For small $L$, the obvious deviation of $\epsilon_{\text {num }}(0)$ and $\epsilon_{\text {fit }}(0)$ 


\begin{tabular}{|l|l|l|l|l|l|l|}
\hline$m$ & $a_{1}$ & $a_{2}$ & $a_{3}$ & $d_{1}$ & $d_{2}$ & $d_{3}$ \\
\hline $10^{-1 / 4}$ & -0.35978636 & 0.32640 & 2.00074 & -0.055407648 & -0.59786 & 2.0001207 \\
$10^{-2 / 4}$ & -0.52340670 & 0.14909 & 2.00060 & -0.087142728 & -0.53736 & 2.0000179 \\
$10^{-3 / 4}$ & -0.5999064788 & 0.071047 & 2.00060 & -0.09949568312 & -0.52648 & 2.0000036 \\
$10^{-1}$ & -0.63561331929 & 0.033594 & 2.00061 & -0.103882817867 & -0.52421 & 2.0000008 \\
\hline
\end{tabular}

Table 2. Fitting parameters for the $L$-dependence of $\epsilon(0)$ and $f$

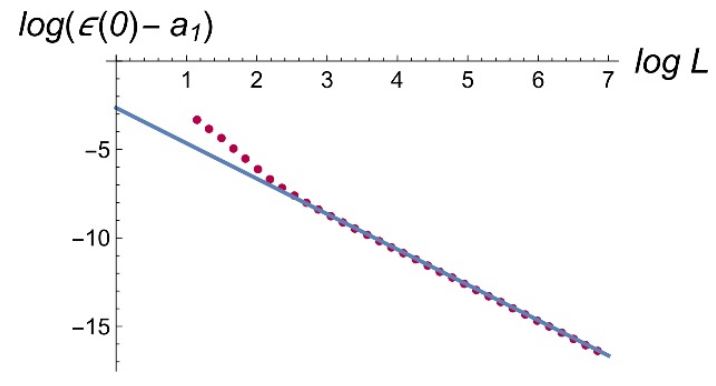

(a)

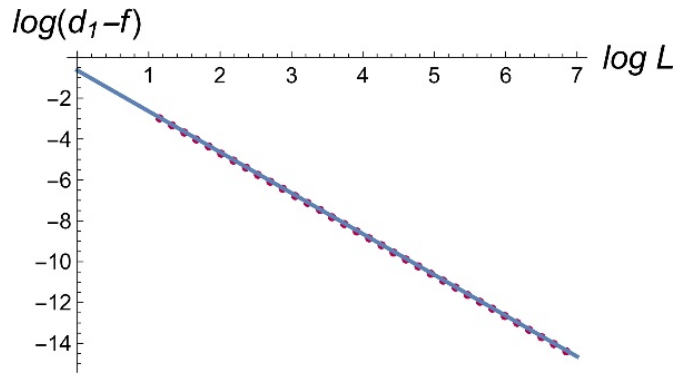

(b)

Figure 2. (a) Plot of $\epsilon(0)$. (b) Plot of $f$. The dots represent the numerical data $\epsilon_{\text {num }}(0), f_{\text {num }}$, whereas the solid lines represent the fitting functions $\epsilon_{\mathrm{fit}}(0), f_{\text {fit }}$.

in figure $2(\mathrm{a})$ is due to the corrections of $\mathcal{O}\left(e^{- \text {(const. }) L}\right)$. The deviation becomes smaller for larger $m / a$, meaning that the constant in the exponent increases as $m / a$. For the above set of the parameters, $y \sim 10^{-2}$ and $L^{-2} \geq 10^{-6}$. Since $e_{n}$ and $k_{n}$ are also small numbers, the truncation of the sums for $a_{1}$ and $d_{1}$ may not affect in observing the $L^{-2}$ scaling as long as the terms up to $\mathcal{O}\left(y^{3}\right)$ are included.

By similar fittings for other $m$ with other parameters fixed, we obtain $a_{j}, d_{j}$ in table 2. They are again consistent with the values in table 1 . If higher order terms in $y$ are included in evaluating these values, we find a better agreement between table 1 and 2 . Relative corrections for $a_{2}, d_{2}$ are larger than those for $a_{1}, d_{1}$. All in all, we conclude that the scaling (3.8) and the $L$-dependence of the expansions are consistent with the numerical results.

\subsection{Dependence on $m / a$}

Next, we would like to check the expansions (3.35), (3.37), (3.43) and (3.44), (3.45). For this purpose, we have solved the original TBA equations (2.12) and evaluated the free energy (2.16) numerically. The parameters are set as $a=4 / 7, m / a=6 / 5-k / 100(k=$ $0, \ldots, 115), L=10^{3}$, and others are the same as in the previous subsection. Though the expansions are valid for $m / a<1$, we include $m>a$ for reference. It is not easy to determine many coefficients in the expansions by data fitting. Instead, we check below if the numerical data are well approximated by the expansions in the previous section.

Figure 3(a) is a plot of $\epsilon(0)$. The numerical data $\epsilon_{\text {num }}(0)$ (dots) are well approximated by $\epsilon^{(6)}(0)$ (solid line), where $\epsilon^{(k)}(\theta)$ is the expansion (3.44) truncated at $n=k$. If other $\epsilon^{(k)}(k \geq 1)$ were plotted, they would be almost degenerate in the figure; $\epsilon^{(1)}(0)$ is already 


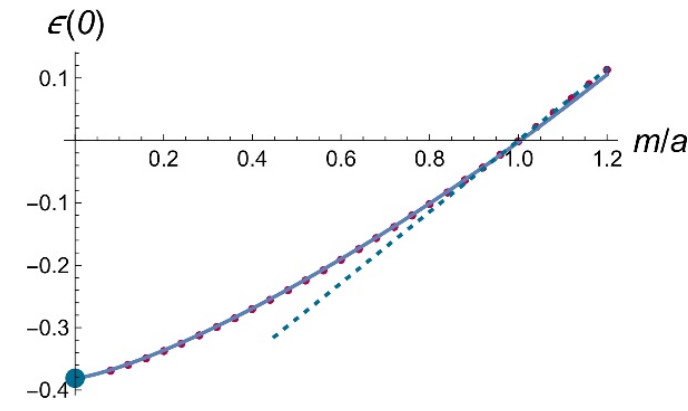

(a)

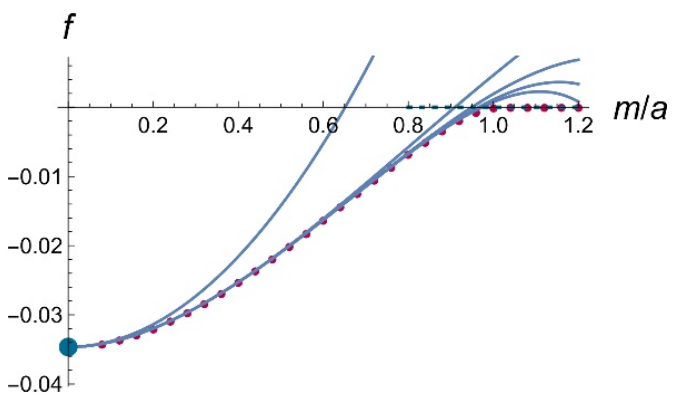

(b)

Figure 3. (a) Plot of $\epsilon(0)$. The dots represent the numerical data $\epsilon_{\text {num }}(0)$, whereas the solid line represents the expansion $\epsilon^{(6)}(0)$. The blob indicates the UV value $\epsilon_{\mathrm{UV}}$. The dotted line represents $\epsilon_{\mathrm{IR}}(0)$. (b) Plot of $f$. The dots represent the numerical data $f_{\text {num }}$, whereas the solid lines represent the expansions $f^{(k)}(0 \leq k \leq 4)$ from the top to the bottom. The blob indicates the UV value $f_{\mathrm{UV}}$. The data converge to $f_{\mathrm{IR}}=0$ (dotted line) as $m / a \rightarrow 1$.

a good approximation because $\left|e_{j} / e_{1}\right| \ll 1(1<j)$. As $m / a \rightarrow 0$, they approach the value in the UV limit $\epsilon_{\mathrm{UV}}=L^{-1} \log \left(e^{-2 A / 3}+e^{-4 A / 3}\right) \approx-0.381$ (blob). As $m / a \rightarrow 1$, the data approach the asymptotic form $\epsilon_{\mathrm{IR}}(0)=-a+m$ in the IR limit (dotted line). See (2.21) and (2.23). One can also check that $\epsilon_{\text {num }}(0)-\epsilon^{(k-1)}(0)$ scales as $(m / a)^{4 k / 3}$, and that it is saturated as $(m / a)^{4 / 3} L^{-2}$ for small enough $m / a$. A similar analysis is possible also for $\tilde{\epsilon}(0)$. including the $\mathcal{O}\left(L^{-2}\right)$ term.

Figure $3(\mathrm{~b})$ is a plot of $f$. The numerical data $f_{\text {num }}(\operatorname{dots})$ are well approximated by $f^{(k)}$ $(k=0,1,2,3,4)$ (solid lines from the top to the bottom), where $f^{(k)}$ is the expansion (3.45) truncated at $n=k$. As $m / a \rightarrow 0$, they approach the value in the UV limit $f_{\mathrm{UV}}=$ $-\left(\frac{\pi}{6}+\frac{A^{2}}{3 \pi}\right) / L^{2} \approx-0.0346$ (blob). As $m / a \rightarrow 1$, the numerical data converge to the value in the IR (free field) limit $f_{\mathrm{IR}}=0$ (dotted line). One can also check that $f_{\text {num }}-f^{(k-1)}$ scales as $(m / a)^{8 k / 3}$, and that it is saturated as $L^{-2}$ without any power of $m / a$ for small enough $m / a$. These observations are again consistent with the results in the previous section.

\section{Analytic expansion of 6-point remainder function}

Having checked the validity of the expansions and the linearlization against numerical data, we now apply the expansions to the strong-coupling amplitudes. In the following, we focus on the leading expansions in $1 / A^{2}$, though it is straightforward to include the subleading $\mathcal{O}\left(1 / L^{2}\right)$ terms as discussed in section 3 . It is thus understood that the equations are valid up to relative corrections, if any, which are exponentially small in $A$ or of $\mathcal{O}\left(A^{-2}\right)$.

\subsection{Cross-ratios}

Let us first discuss how the cross-ratios change according to the changes in the parameters of the TBA equations. From the expansions (3.44) which are valid for any $\operatorname{Im} \theta$, one can obtain $u_{j}$ by substituting these into (2.18). Alternatively, one can consider the variables 


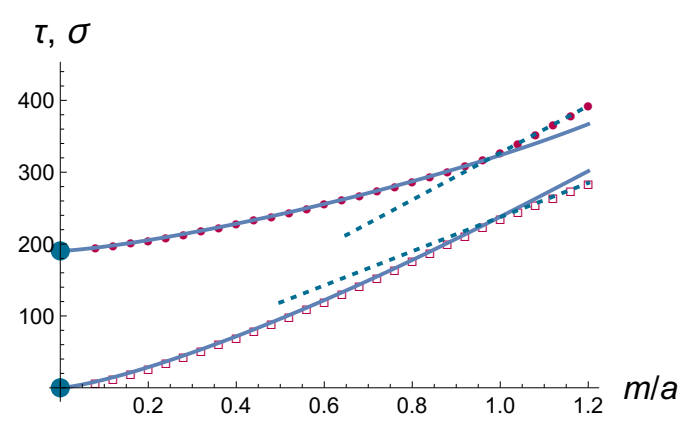

(a)

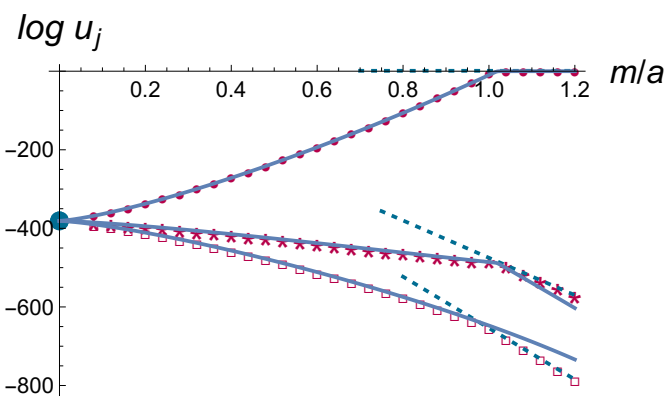

(b)

Figure 4. (a) Plot of $\tau$ and $\sigma$. The numerical data of $\tau$ and $\sigma$ are plotted by $\cdot$ and $\square$, respectively. The solid lines represent the analytic expansions from those of $\varepsilon, \tilde{\varepsilon}$. The blobs $(\bullet)$ represent their $\mathrm{UV}$ values, $\tau_{\mathrm{UV}}, \sigma_{\mathrm{UV}}$. The dotted lines represent their asymptotic forms, $\tau_{\mathrm{IR}}, \sigma_{\mathrm{IR}}$. The constant $\phi=-A$ is omitted. (b) Plot of the cross-ratios $u_{j}$. The numerical data of $u_{1}, u_{2}, u_{3}$ are plotted by $\cdot, \square$ and $*$, respectively. The solid lines represent the analytic expressions from those of $\varepsilon, \tilde{\varepsilon}$. The blob (•) represents the UV value. The dotted lines represent the asymptotic forms in the IR region.

in (2.19) parametrizing the cross-ratios. From (2.13) and (2.19), they are given by

$$
\phi=-A, \quad \tau=\frac{1}{2} \tilde{\varepsilon}\left(-\frac{i \pi}{4}-i \varphi\right), \quad \sigma=A+\varepsilon(-i \varphi)-\frac{1}{2} \tilde{\varepsilon}\left(-\frac{i \pi}{4}-i \varphi\right) .
$$

For small $m / a$, the pseudo energies $\epsilon, \tilde{\epsilon}$ approach their UV values in (2.21), and hence

$$
\tau \rightarrow \tau_{\mathrm{UV}}:=\frac{A}{3}, \quad \sigma \rightarrow \sigma_{\mathrm{UV}}:=0
$$

For $m / a \gtrsim 1$, the pseudo energies approach their IR forms (2.23), and hence

$$
\tau \rightarrow \tau_{\mathrm{IR}}:=\frac{M}{\sqrt{2}} \cos \left(\frac{\pi}{4}+\varphi\right), \quad \sigma \rightarrow \sigma_{\mathrm{IR}}:=\frac{M}{\sqrt{2}} \cos \left(\frac{\pi}{4}-\varphi\right) .
$$

Figure 4(a) is a plot of $\tau$ and $\sigma$ for the same parameters of the TBA equations as in subsection 4.2. The points (.) and the boxes ( $\square$ ) represent the numerical data of $\tau$ and $\sigma$, respectively. The solid lines represent the analytic expansions from those of $\varepsilon, \tilde{\varepsilon}$ in (3.44). The UV values $\tau_{\mathrm{UV}} \sim 190$ and $\sigma_{\mathrm{UV}}=0$ are denoted by the blobs $(\bullet)$. The asymptotic forms in the IR, $\tau_{\mathrm{IR}} \sim 327 \cdot(\mathrm{m} / \mathrm{a}), \sigma_{\mathrm{IR}} \sim 238 \cdot(\mathrm{m} / \mathrm{a})$, are plotted by the dotted lines. The other variable is a constant, $\phi=-A=-(4 / 7) \cdot 10^{3} \sim-571$. As $\varphi \rightarrow-\pi / 4$, the IR form $\sigma_{\mathrm{IR}}$ is flattened, which corresponds to the collinear limit.

In the following, we consider the case where $-\pi / 4<\varphi<0$, unless otherwise stated, as in the above. Other cases are analyzed similarly. In this case, we find that

$$
e^{A} \gg e^{\tau} \gg e^{\sigma} \gg 1
$$

generically for $0 \leq m / a$. The exceptions are $e^{\sigma} \sim \mathcal{O}(1)$ for small $m / a$ or $\varphi \rightarrow-\pi / 4$, and $e^{\tau-\sigma} \sim \mathcal{O}(1)$ for $\varphi \sim 0$ and $m / a \gtrsim 1$. 
The behavior of $(\tau, \sigma, \phi)$ is translated into that of $u_{j}$ by using the relations (2.20). First, it follows from (5.2) that

$$
u_{1}, u_{2}, u_{3} \rightarrow u_{\mathrm{UV}}:=e^{-\frac{2}{3} A} \ll 1
$$

as $m / a \rightarrow 0$. To estimate $u_{j}$ for other $m / a$, we note that $\sigma+\tau$ increases as $m / a$ and approaches $M \cos \varphi$. Thus,

$$
u_{1} \sim e^{\sigma+\tau-A}, \quad u_{2} \sim e^{-2 \tau}, \quad u_{3} \sim e^{-\sigma+\tau-A},
$$

for $m / a<1$, where the expansions (3.35) and (3.37), or (3.44) are valid. Their product is still a constant $u_{1} u_{2} u_{3} \sim e^{-2 A}$ approximately. As $M$ reaches $A / \cos \varphi$, the leading term in $u_{3}$ changes and hence

$$
u_{1} \sim 1-e^{A-\tau-\sigma}, \quad u_{2} \sim e^{-2 \tau}, \quad u_{3} \sim e^{-2 \sigma}
$$

for $m / a>1 / \cos \varphi$, where the asymptotic forms (5.3) are valid. The cross-ratios $u_{j}$ are kept small as $m / a$ is varied.

Figure $4(\mathrm{~b})$ is a plot of $u_{j}$. The points $(\cdot)$, boxes $(\square)$ and asterisks $(*)$ represent the numerical data of $u_{1}, u_{2}$ and $u_{3}$, respectively, for the same parameters as above. They are obtained by substituting the numerical solution of (2.12) into the full expressions of the cross-ratios (2.20) with (5.1). The solid lines represent the corresponding analytic expansions from those of $\varepsilon, \tilde{\varepsilon}$ in $(3.44)$. The blob $(\bullet)$ represents the value in the UV limit $\hat{u}_{\mathrm{UV}} \sim u_{\mathrm{UV}}=e^{-2 A / 3}$. The dotted lines represent the asymptotic forms obtained from $\tau_{\mathrm{IR}}$ and $\sigma_{\mathrm{IR}}$. Though the expansions are valid for $m / a<1$, the solid lines approximate the numerical data even in the IR region. They are further approximated by (5.4) for $m / a<1 / \cos \varphi$ and by (5.5) for $m / a>1 / \cos \varphi$.

The trajectory of $u_{j}$ is also summarized in figure 1 in section 2: the UV point $\left(u_{1}, u_{2}, u_{3}\right)=\left(\hat{u}_{\mathrm{UV}}, \hat{u}_{\mathrm{UV}}, \hat{u}_{\mathrm{UV}}\right)$ for $m / a=0$ is denoted by the lower blob in the figure. This corresponds to the regular polygonal limit. As $m / a$ increases, $u_{j}$ moves along the yellow surface $u_{1} u_{2} u_{3} \sim e^{-2 A}$, until $m / a$ reaches $1 / \cos \varphi$. The expansions (3.35) and (3.37), or (3.44) are valid there for $m / a<1$. For larger $m / a$, the cross-ratios arrive at the IR point $\left(u_{1}, u_{2}, u_{3}\right)=(1,0,0)$ denoted by the upper blob. This corresponds to a soft limit. The transition from (5.4) to (5.5) happens in a small region $\delta(m / a) \sim \mathcal{O}(1 / A)$. This rapid change explains the apparent bends in figure 4(b): though some derivatives of the plots might look discontinuous around $m / a \sim 1$, that is not the case.

In terms of $(\tau, \sigma, \phi)$, these two regimes are smoothly connected. This is also confirmed by the fact that the yellow surface in figure 1 asymptotes for large $A$ to $u_{1} u_{2} u_{3}=0$ which includes the IR end point. Sending $\varphi \rightarrow-\pi / 4$, one can adjust $u_{3} \sim 1 /\left(1+e^{2 \sigma}\right)$ for large enough $m / a$, so that the end point becomes $\left(1-u_{3}, 0, u_{3}\right)$ with non-vanishing $u_{3}$. This corresponds to a collinear limit. Thus, by changing $M$ and $\varphi$ (including generic $\varphi$ ), the trajectories of $u_{j}$ for large $A$ form a surface ("cap") which is close to $u_{1} u_{2} u_{3}=e^{-2 A}$ but ends on the triangle of the collinear/soft limit. 


\subsection{Expansion of 6-point remainder function}

We now focus on the region where $m / a<1$ and the expansions of (3.35) and (3.37), or (3.44) are valid. The other region with $m / a \gtrsim 1$ can be discussed by using the asymptotic IR forms. In this region, $u_{j}$ are small, and the dilogarithm function in $\Delta A_{\mathrm{BDS}}$ in (2.17) is approximated by $\operatorname{Li}_{2}\left(1-u_{j}^{-1}\right) \sim-\frac{1}{2} \log ^{2} u_{j}-\pi^{2} / 6$. Substituting the expansions (3.44) and omitting the terms relatively of $\mathcal{O}\left(A^{-2}\right)$ from $\pi^{2} / 6$ above, we find the $(\mathrm{m} / \mathrm{a})$-expansion of $\Delta A_{\mathrm{BDS}}$ :

$$
\Delta A_{\mathrm{BDS}}=A^{2} \sum_{n=0}^{\infty} \beta_{n} y^{n / 2}
$$

with

$$
\begin{array}{ll}
\beta_{0}=\frac{1}{6}, & \beta_{1}=0, \\
\beta_{2}=\frac{3 \pi}{\Gamma^{2}\left(-\frac{1}{6}\right) \Gamma^{2}\left(\frac{2}{3}\right)}, & \beta_{3}=- \\
\beta_{4}=\frac{\pi}{48 \Gamma^{2}\left(\frac{1}{6}\right) \Gamma^{2}\left(\frac{1}{3}\right)}+\frac{2^{\frac{1}{3}} \pi^{\frac{3}{2}} \Gamma\left(\frac{1}{6}\right)}{\Gamma^{4}\left(-\frac{1}{6}\right) \Gamma^{2}\left(\frac{2}{3}\right)}, & \beta_{5}=- \\
\beta_{6}=-\frac{17 \cos (8 \varphi)}{2^{9} 3^{3} \sqrt{3} \pi}+\frac{19 \Gamma^{2}\left(\frac{1}{3}\right) \Gamma^{6}\left(\frac{7}{6}\right)}{128 \times 2^{\frac{1}{3}} \pi^{4} \Gamma^{2}\left(\frac{5}{6}\right)}, &
\end{array}
$$

for small n. $\Delta A_{\mathrm{BDS}}$ is symmetric under $\varphi \rightarrow \varphi+2 \cdot(\pi / 4)$, which permutes the three cross-ratios $u_{j}$. This symmetry strongly constrains the expansion, and only the terms with $\cos \frac{4 n}{3} \varphi(n \in 3 \mathbb{Z})$ should survive [37]. From the expansions (3.44), such terms are not possible at $\mathcal{O}\left(y^{1 / 2}\right)$. The above expansion indeed satisfies this constraint with vanishing $\beta_{1}$.

Combining the other terms, we obtain the expansion of the 6-point remainder function,

$$
\begin{aligned}
R_{6} & =\Delta A_{\mathrm{BDS}}-A_{\text {periods }}-A_{\text {free }} \\
& =A^{2} \sum_{n=0}^{\infty} \beta_{n} y^{n / 2}-\frac{A^{2}}{\pi} \sum_{n=0}^{\infty} k_{n} y^{n},
\end{aligned}
$$

where $\Delta A_{\mathrm{BDS}}$ is given in (5.6), $A_{\text {periods }}=M^{2} / 4$ as in (2.17), and $A_{\text {free }}=-L^{2} f$ with $f$ given in (3.45). The coefficients $\beta_{n}$ and $k_{n}$ for small $n$ are given in (5.7) and (3.46), respectively, whereas $y \sim(M / A)^{\frac{8}{3}}$ is defined in (3.20). The bulk term $-M^{2} / 4$ in $A_{\text {free }}$ canceled $A_{\text {periods. }}$. The expansion is valid for $m / a<1$ and large $A=a L, M=m L$ up to the relative corrections which are exponentially small in $A$ or of $\mathcal{O}\left(A^{-2}\right)$.

Figure $5(\mathrm{a})$ is a plot of the 6-point remainder function for the parameters as in subsection 4.2. The points $(\cdot)$ represent the numerical data from the original TBA equations (2.12). The solid line represents the expansion of $R_{6}$ given above which includes terms up to $(m / a)^{8}$. The blob $(\bullet)$ is the value in the UV limit $R_{\mathrm{UV}}$ in (2.22). The dotted line represents the value in the IR limit $R_{\mathrm{IR}}$ in (2.24).

The expansion well approximates the numerical data over the region from $m / a=0$ to $m / a=1$, and interpolates the UV and the IR regime. The relative correction of the expansion to the numerical value is $2.6 \times 10^{-5}$ at $m / a=0.05$, and $2.5 \times 10^{-2}$ at $m / a=0.9$. 


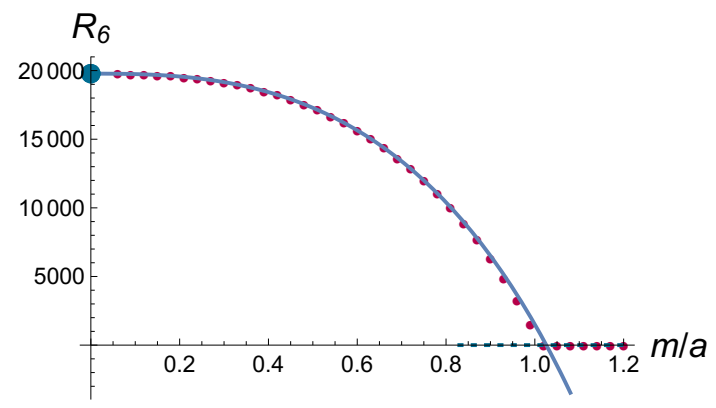

(a)

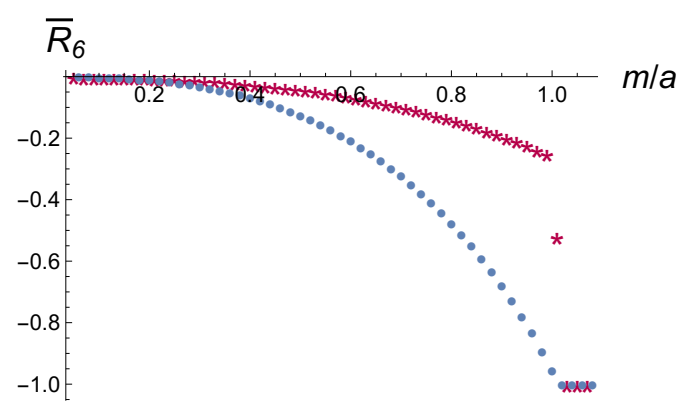

(b)

Figure 5. (a) Plot of 6-point remainder function at strong coupling. The points represent the numerical data. The solid line represents the analytic expansion. The blob $(\bullet)$ represents $R_{6, \mathrm{UV}}$. The dotted line represents $R_{6, \mathrm{IR}}$. (b) Plot of 6-point rescaled remainder functions. The points $(\cdot)$ and the asterisks $(*)$ represent the numerical data at strong coupling, $\bar{R}_{6}^{\text {strong }}$, and at 2 loops, $\bar{R}_{6}^{2 \text {-loop }}$, respectively.

The former is attributed to the $\mathcal{O}\left(L^{-2}\right)$ correction in our expansions, whereas the latter is to the truncation of the expansions. By including higher order terms, one obtains a better approximation to the numerical data up to $m / a \sim 1$. If we use the expression of $\Delta A_{\mathrm{BDS}}$ by substituting the expansions (3.44) into (2.17) (without expanding in $y^{n / 2}$ ), higher order terms are partially incorporated and its IR behavior for $m / a \gtrsim 1$ is improved.

\subsection{Comparison with 2-loop results}

In $[40,41,44]$, the remainder functions corresponding to the minimal surfaces in $\mathrm{AdS}_{3}$ were compared at strong coupling and at 2 loops $[17,18]$ by changing the mass parameters of the TBA system with other parameters fixed. It was found that they are close to each other (but different) after appropriately rescaled/normalized. Such an observation was also made for the $\mathrm{AdS}_{4}$ case [39]. In the present 6-point case with the general ( $\left.\mathrm{AdS}_{5}\right)$ kinematics, the comparison was extended and the similarity was confirmed up to 4 loops [38] based on the perturbative results $[19,20]$. The comparisons for other kinematics are found in $[19,20]$. In this subsection, we compare the remainder functions at strong coupling and at 2 loops for the cross-ratios discussed in the previous subsections.

For this purpose, we introduce the remainder function at strong coupling which is rescaled by its UV and IR values,

$$
\bar{R}_{6}^{\text {strong }}:=\frac{R_{6}-R_{6, \mathrm{UV}}}{R_{6, \mathrm{UV}}-R_{6, \mathrm{IR}}},
$$

with $R_{6, \mathrm{UV}}, R_{6, \mathrm{IR}}$ given in (2.22) and (2.24), respectively. For the remainder function in perturbation $R_{6}^{\text {pert }}=\sum \lambda^{\ell} R_{6}^{(\ell)}$ where $\lambda$ is the 't Hooft coupling, the rescaled remainder functions $\bar{R}_{6}^{\ell \text {-loop }}$ are defined similarly. In particular at 2 loops,

$$
\bar{R}_{6}^{2 \text {-loop }}:=\frac{R_{6}^{(2)}-R_{6, \mathrm{UV}}^{(2)}}{R_{6, \mathrm{UV}}^{(2)}-R_{6, \mathrm{IR}}^{(2)}},
$$


where $R_{6, \mathrm{UV}}^{(2)}=R_{6}^{(2)}\left(\hat{u}_{\mathrm{UV}}, \hat{u}_{\mathrm{UV}}, \hat{u}_{\mathrm{UV}}\right), R_{6, \mathrm{IR}}^{(2)}=0$. The analytic expression of $R_{6}^{(2)}$ is given [17, 18] by

$$
\begin{aligned}
R_{6}^{(2)}\left(u_{1}, u_{2}, u_{3}\right)= & \sum_{i=1}^{3}\left(L_{4}\left(x_{i}^{+}, x_{i}^{-}\right)-\frac{1}{2} \operatorname{Li}_{4}\left(1-u_{i}^{-1}\right)\right)-\frac{1}{8}\left(\sum_{i=1}^{3} \operatorname{Li}_{2}\left(1-u_{i}^{-1}\right)\right)^{2} \\
& +\frac{1}{24} J^{4}+\frac{\pi^{2}}{12} J^{2}+\frac{\pi^{4}}{72}
\end{aligned}
$$

where

$$
x_{i}^{ \pm}=u_{i} x^{ \pm}, \quad x^{ \pm}=\frac{u_{1}+u_{2}+u_{3}-1 \pm \sqrt{\Delta}}{2 u_{1} u_{2} u_{3}}, \quad \Delta=\left(u_{1}+u_{2}+u_{3}-1\right)^{2}-4 u_{1} u_{2} u_{3},
$$

and

$$
\begin{aligned}
L_{4}\left(x^{+}, x^{-}\right) & =\frac{1}{8 ! !} \log ^{4}\left(x^{+} x^{-}\right)+\sum_{m=0}^{3} \frac{(-1)^{m}}{(2 m) ! !} \log ^{m}\left(x^{+} x^{-}\right) \times\left(\ell_{4-m}\left(x^{+}\right)+\ell_{4-m}\left(x^{-}\right)\right), \\
\ell_{n}(x) & =\frac{1}{2}\left(\operatorname{Li}_{n}(x)-(-1)^{n} \operatorname{Li}_{n}\left(x^{-1}\right)\right), \\
J & =\sum_{i=1}^{3}\left(\ell_{1}\left(x_{i}^{+}\right)-\ell_{1}\left(x_{i}^{-}\right)\right) .
\end{aligned}
$$

By definition, $\bar{R}_{6}^{\text {strong }}, \bar{R}_{6}^{2 \text {-loop }} \rightarrow 0$ in the UV limit, and $\bar{R}_{6}^{\text {strong }}, \bar{R}_{6}^{2 \text {-loop }} \rightarrow-1$ in the IR limit.

Figure $5(\mathrm{~b})$ is a plot of the rescaled remainder functions at strong coupling and at 2 loops for the cross-ratios given in section 5.1. The points $(\cdot)$ represent the numerical data of $\bar{R}_{6}^{\text {strong }}$ which are obtained from the numerical solution of (2.12). The asterisks $(*)$ represent $\bar{R}_{6}^{2 \text {-loop }}$ which are obtained by substituting into (5.8) the same numerical values of the cross-ratios.

To avoid loss of significant digits in the numerical evaluation of $R_{6}^{(2)}$, we have made an approximation,

$$
x^{+} \sim \frac{1}{u_{1}+u_{2}+u_{3}-1}, \quad x^{-} \sim \frac{u_{1}+u_{2}+u_{3}-1}{u_{1} u_{2} u_{3}},
$$

which is valid for $\left(1-u_{1}-u_{2}-u_{3}\right)^{2} \gg u_{1} u_{2} u_{3}$, and hence both for (5.4) and (5.5) up to exponentially small terms in $A$. By using the analytic expansion of $R_{6}$ at strong coupling, one can draw a plot of $\bar{R}_{6}^{\text {strong }}$ which well approximates the data points as in figure $5(\mathrm{a})$. Since the cross-ratios $u_{j}$ are well approximated by the analytic expansions, substituting them into $R_{6}^{(2)}$ in (5.8) also gives a plot which approximates the data points as well.

The behaviors of $\bar{R}_{6}^{\text {strong }}$ and $\bar{R}_{6}^{2 \text {-loop }}$ are rather different for large $m / a$, where $\bar{R}_{6}^{\text {strong }}$ decreases faster than $\bar{R}_{6}^{2 \text {-loop }}$. This is in contrast to the results mentioned above by varying the mass parameters with others fixed.

\section{Conclusions}

Through the gauge-string duality, the MHV amplitudes of $\mathcal{N}=4$ SYM at strong coupling are obtained by solving auxiliary integral equations of the TBA type. In this paper, we 
considered the limit where chemical potentials in addition to masses are large, and hence the TBA equations are linearized. Large chemical potentials, together with large masses, thus provide another useful limit. In particular, the linearized TBA equations for the 6point amplitudes are solved analytically according to [43] as expansions to any order in terms of the ratio of the mass and the chemical potential $M / A$. The relative corrections in the linearization are exponentially small in $A$ or of $\mathcal{O}\left(1 / A^{2}\right)$. The inverse power corrections are analyzed by extending the analysis in [43], and we obtained the explicit forms of the leading corrections. We checked that our results agree with numerical solutions.

Assured that the linearization gives a controlled approximation of the original TBA equations for large $A$ and $M$, we derived analytic expansions of the 6-point MHV amplitudes from the solution of the linearized TBA equations. The expansion is again valid to any order up to corrections exponentially small in $A$ or of $\mathcal{O}\left(A^{-2}\right)$. As $M$ is varied with $A \gg 1$ and the phase $\varphi$ fixed, the three cross-ratios $u_{j}$ of the momenta of scattering particles are kept small and change from the equal value $u_{j} \sim e^{-2 A / 3}$ in the UV regime to those in the soft/collinear limits in the IR regime. The amplitudes are well described by the expansion over the corresponding kinematic region.

We also compared the 6-point rescaled remainder functions at strong coupling and at 2 loops along the trajectory of $u_{j}$ mentioned above. We observed that they are rather different, in contrast to the cases where similarities are observed between the strong-coupling results and the perturbative results $[19,20,38-41,44]$. This implies that the kinematic region of the small cross ratios provides a useful probe to study structural differences of the strong-coupling and the perturbative results. For example, since the perturbative results share the property that they are controlled by the transcendentality and the associated symbol [17, 19-21], the difference from the strong-coupling case may persist for higher loops. The actual comparison with higher-loop results in [19-21] is thus an interesting future problem.

The extension of our analysis to the general $n$-point amplitudes would be an important future direction. For $n>6$, the TBA system has more than one mass scale, and the generalization of the work [43] is not straightforward. This is closely related to the problem of finding the exact mass-coupling relation, i.e. the relation between the physical mass and the coupling in the Lagrangian, for multi-scale integrable models. As mentioned in section 2 , this problem has been solved $[58,59]$ for a simple multi-scale integrable model, i.e. the $s u(3)_{2} / u(1)^{2}$ HSG model, which is relevant for the 10-point amplitudes for two-dimensional kinematics. The issue of the multi-scales has been overcome there by comparing the picture of the conformal perturbation on the UV side and that of the form-factor bootstrap on the IR side.

By adjusting the phase $\varphi$, the IR end point of the trajectory of $u_{j}$ can be set to the point of the collinear limit, around which the OPE expansion at finite coupling is derived $[5,36]$. It would be of interest to consider if our expansion at strong coupling provides useful data for the OPE expansion. 


\section{Acknowledgments}

Y.S. would like to thank Zoltan Bajnok for useful discussions and for sharing his notes on the linearization of TBA equations, and Davide Fioravanti for useful discussions. This work is supported in part by JSPS Grant-in-Aid for Scientific Research 24540248, 15K05043, 15K05208, 16F16735, 17K05406, 18H01141, 18K03452 and 18K03643, and Japan-Hungary Research Cooperative Program from Japan Society for the Promotion of Science (JSPS).

\section{A Evaluation of $\tilde{\epsilon}$ from direct integrals}

In this appendix, we evaluate the expansion of $\tilde{\epsilon}$ in (3.19) by plugging that of $\epsilon$ (3.18) into (3.7). For this purpose, we note the formula,

$$
\int d x \frac{e^{c x}}{\cosh (a x+b)}=\frac{2}{a+c} e^{(a+c) x+b}{ }_{2} F_{1}\left(1, \frac{a+c}{2 a} ; 1+\frac{a+c}{2 a} ;-e^{2(a x+b)}\right) .
$$

This is checked by the expansion of the hypergeometric function,

$$
{ }_{2} F_{1}(1, \beta ; \beta+1 ; z)=\sum_{n=0}^{\infty} \frac{\beta z^{n}}{\beta+n}=: \Phi_{\beta}(z),
$$

for $|z|<1$, and its analytic continuation in $z$, from which we find

$$
\Phi_{\beta}(z)+\frac{z}{\beta} \Phi_{\beta}^{\prime}(z)=\frac{1}{1-z} .
$$

Taking the derivative of the right hand side of (A.1) with the help of (A.2) and setting $z=$ $-e^{2(a x+b)}, \beta=(a+c) / 2 a$, we obtain the formula. Furthermore using the inversion formula,

$$
{ }_{2} F_{1}(a, b ; c ; z)=\frac{\Gamma(b-a) \Gamma(c)}{\Gamma(b) \Gamma(c-a)}(-z)^{-a}{ }_{2} F_{1}\left(a, a-c+1 ; a-b+1 ; z^{-1}\right)+(a \leftrightarrow b),
$$

and

one finds

$$
{ }_{2} F_{1}(a, b ; b ; z)=(1-z)^{-a}, \quad \Gamma(1-z) \Gamma(z)=\frac{\pi}{\sin \pi z},
$$

$$
\begin{aligned}
\int_{-B}^{B} d \theta^{\prime} \frac{e^{c \theta^{\prime}}}{\cosh 2\left(\theta^{\prime}-\theta\right)}= & \frac{\pi}{2} \frac{e^{c \theta}}{\sin \pi \beta}-\frac{e^{-2 \theta-4 \beta B}}{2 \beta} \Phi_{\beta}\left(-e^{-4(B+\theta)}\right) \\
& -\frac{e^{2 \theta-4(1-\beta) B}}{2(1-\beta)} \Phi_{1-\beta}\left(-e^{-4(B-\theta)}\right),
\end{aligned}
$$

with $\beta=(2+c) / 4$. Using this, we get

$$
\begin{aligned}
I_{n}(B, \theta):=\int_{-B}^{B} \frac{d \theta^{\prime}}{2 \pi} K_{2}\left(\theta-\theta^{\prime}\right) \cosh \left(\frac{4 n}{3} \theta^{\prime}\right) \\
=(-1)^{n} \cosh \left(\frac{4 n}{3} \theta\right)-\frac{\sqrt{2}}{2 \pi} \sum_{\ell, \ell^{\prime}= \pm 1} \frac{e^{-\left(2+s_{n}\right) B}}{2+s_{n}} \\
\times \\
\times\left[e^{(2+\ell) \theta} \Phi_{\frac{2+s_{n}}{4}}\left(-e^{-4(B-\theta)}\right)+(\theta \rightarrow-\theta)\right],
\end{aligned}
$$


with $s_{n}=\frac{4 n}{3} \ell^{\prime}+\ell$. From (3.18) and (3.7), we thus find

$$
\tilde{\epsilon}(\theta)=\sqrt{2} m \cosh \theta+\frac{2 a}{3} I_{0}(B, \theta)+\sum_{n>0} c_{n \neq 0(\bmod 3)} c_{n}(B, \theta) .
$$

Comparing this with (3.19), we observe that the summation over $\Phi_{\left(2+s_{n}\right) / 4}$ in $I_{n}$ cancels the driving term $\sqrt{2} m \cosh \theta$, which leaves the first terms $(-1)^{n} \cosh (4 n \theta / 3)$ in accordance with the periodicity required from the Y-system. These fractional powers of $e^{\theta}$ appeared by summing up integral powers of $e^{\theta}$ in $\Phi_{\beta}$ and analytically continuing it by the inversion (A.3).

\section{B Estimation of $\epsilon^{\prime}(B)$}

We consider the evaluation of $\epsilon^{\prime}(B)$ (and $\xi_{1}$ ), which is necessary for $\kappa_{n m}$ in $(3.27)$ and $q^{(1)}$ in (3.33) for example. For the derivative of (3.10) with respect to $\theta$, we obtain

$$
D_{\epsilon^{\prime}}(\theta)=\int_{-\infty}^{\infty} \tilde{K}\left(\theta-\theta^{\prime}\right) \epsilon_{B}^{\prime}\left(\theta^{\prime}\right) d \theta^{\prime}
$$

where

$$
D_{\epsilon^{\prime}}(\theta)=y(\theta)-y(-\theta), \quad y(\theta)= \begin{cases}-\frac{m}{2} \mathrm{e}^{\theta} & \theta<B \\ x(\theta) & \theta>B .\end{cases}
$$

Here the terms like $\Delta_{1}^{\prime}(\theta)$ are neglected as they are higher order terms.

Set

$$
\tau(w)=\widehat{K}_{-}(w) \widehat{y}(w) \mathrm{e}^{-i w B}
$$

By repeating the same argument as in section 3, we obtain an integral equation for $\tau(w)$,

$$
\begin{aligned}
\tau(w) & =D_{\tau}(w)-\int_{-\infty}^{\infty} \frac{\mathrm{e}^{2 i w^{\prime} B}}{w+w^{\prime}+i \varepsilon} \alpha\left(w^{\prime}\right) \tau\left(w^{\prime}\right) \frac{d w^{\prime}}{2 \pi i}, \\
D_{\tau}(w) & =i \frac{m e^{B} \widehat{K}_{+}(-i)}{2(w-i)}
\end{aligned}
$$

The solution is formally given by

$$
\tau(w)=i \frac{m e^{B} \widehat{K}_{-}(i)}{2(w-i)}-\sum_{n \geq 1, n \neq 0(\bmod 3)} \frac{\mathrm{e}^{-\frac{8}{3} n B}}{w+\frac{4}{3} n i} \alpha_{n} \tau\left(\frac{4}{3} n i\right),
$$

where $\alpha_{n}=\operatorname{res}_{w=\frac{4}{3} n i} \alpha(w)$.

Thus we obtain a set of algebraic equations for $\hat{\tau}_{\ell}(\ell \in \mathbb{N})$,

$$
\begin{aligned}
\widehat{\tau}_{\ell} & =\frac{1}{\frac{4 \ell}{3}-1}-\sum_{n \geq 1, n \neq 0(\bmod 3)} \frac{q^{n}}{\ell+n} \frac{(-1)^{n}}{(n-1) ! n !} \frac{\Gamma\left(1+\frac{n}{3}\right) \Gamma\left(\frac{1}{2}+\frac{2 n}{3}\right)}{\Gamma\left(1-\frac{n}{3}\right) \Gamma\left(\frac{1}{2}-\frac{2 n}{3}\right)} \widehat{\tau}_{n}, \\
\widehat{\tau}_{\ell} & =\tau\left(\frac{4}{3} i \ell\right) \frac{2 \mathrm{e}^{-B}}{m \widehat{K}_{-}(i)} .
\end{aligned}
$$


From this, one obtains $\widehat{\tau}_{m}$ as a power series in $q, \widehat{\tau}_{\ell}=\frac{1}{\frac{4 \ell}{3}-1}+\mathcal{O}(q)$. By substituting them into (B.1) we obtain $\tau(w)$. Then $\widehat{\epsilon}_{B}^{\prime}(w)$ is evaluated by

$$
\widehat{\epsilon}_{B}^{\prime}(w)=\widehat{K}_{+}(w) \tau(w) \mathrm{e}^{i w B}-\widehat{K}_{-}(w) \tau(-w) \mathrm{e}^{-i w B} .
$$

The inverse Fourier transformation yields $\epsilon_{B}^{\prime}(\theta)$,

$$
\epsilon_{B}^{\prime}(\theta)=\int_{-\infty}^{\infty} \frac{d w}{2 \pi} \mathrm{e}^{i w(B-\theta)} \widehat{K}_{+}(w) \tau(w)-\int_{-\infty}^{\infty} \frac{d w}{2 \pi} \mathrm{e}^{i w(B+\theta)} \widehat{K}_{+}(w) \tau(w) .
$$

In particular, at $\theta=B$, we deform in the first term the finite part of the integration contour to a semi-circle in the lower half plane, and take the large radius limit of the semi-circle. Then, we obtain

$$
\int_{-\infty}^{\infty} \frac{d w}{2 \pi} \widehat{K}_{+}(w) \tau(w)=-\frac{m}{4} \widehat{K}_{-}(i) \mathrm{e}^{B}\left[1+i \sum_{\substack{n \geq 1 \\ n \neq 0(\bmod 3)}} \mathrm{e}^{-\frac{8}{3} n B} \alpha_{n} \widehat{\tau}_{n}\left(1-2 \widehat{K}_{+}\left(-\frac{4}{3} n i\right)\right)\right] .
$$

The first and the second term in the bracket come from the semi-circle, whereas the third from the poles which are picked up in deforming the contour. We have used $\widehat{K}_{+}(w) \rightarrow 1$ for large $|w|$. In the second term in (B.2), we close the integration contour in the upper half plane, to obtain the contributions which cancel the third term in (B.3). Combining these, we find

$$
\epsilon_{B}^{\prime}(B)=-\frac{m}{4} \widehat{K}_{-}(i) \mathrm{e}^{B}\left[1+i \sum_{\substack{n \geq 1 \\ n \neq 0(\bmod 3)}} \mathrm{e}^{-\frac{8}{3} n B} \alpha_{n} \widehat{\tau}_{n}\right]
$$

We note $\epsilon_{B}^{\prime}(B)=\frac{1}{2}\left(\epsilon_{B}^{\prime}\left(B-0^{+}\right)+\epsilon_{B}^{\prime}\left(B+0^{+}\right)\right)=\frac{1}{2} \epsilon_{B}^{\prime}\left(B-0^{+}\right)$, as $\epsilon_{B}^{\prime}(\theta)$ is discontinuous at $|\theta|=B$ and vanishes for $|\theta|>B$. Thus, $\left|\epsilon^{\prime}(B)\right|=\left|\epsilon_{B}^{\prime}\left(B-0^{+}\right)\right|=2\left|\epsilon_{B}^{\prime}(B)\right|$ with $\epsilon_{B}^{\prime}(B)$ in (B.4). Since $B=\mathcal{O}\left(L^{0}\right)$, it is clear that $\epsilon^{\prime}(B)=\mathcal{O}\left(L^{0}\right)$.

Open Access. This article is distributed under the terms of the Creative Commons Attribution License (CC-BY 4.0), which permits any use, distribution and reproduction in any medium, provided the original author(s) and source are credited.

\section{References}

[1] N. Beisert et al., Review of AdS/CFT integrability: an overview, Lett. Math. Phys. 99 (2012) 3 [arXiv: 1012.3982] [INSPIRE].

[2] N. Gromov, V. Kazakov and P. Vieira, Exact spectrum of anomalous dimensions of planar $N=4$ supersymmetric Yang-Mills theory, Phys. Rev. Lett. 103 (2009) 131601 [arXiv: 0901.3753] [INSPIRE].

[3] D. Bombardelli, D. Fioravanti and R. Tateo, Thermodynamic Bethe ansatz for planar AdS/CFT: a proposal, J. Phys. A 42 (2009) 375401 [arXiv:0902.3930] [INSPIRE]. 
[4] G. Arutyunov and S. Frolov, Thermodynamic Bethe ansatz for the $A d S_{5} \times S^{5}$ mirror model, JHEP 05 (2009) 068 [arXiv: 0903.0141] [INSPIRE].

[5] B. Basso, A. Sever and P. Vieira, Spacetime and flux tube S-matrices at finite coupling for $N=4$ supersymmetric Yang-Mills theory, Phys. Rev. Lett. 111 (2013) 091602 [arXiv: 1303.1396] [INSPIRE].

[6] L.F. Alday and J.M. Maldacena, Gluon scattering amplitudes at strong coupling, JHEP 06 (2007) 064 [arXiv: 0705.0303] [INSPIRE].

[7] J.M. Drummond, G.P. Korchemsky and E. Sokatchev, Conformal properties of four-gluon planar amplitudes and Wilson loops, Nucl. Phys. B 795 (2008) 385 [arXiv:0707.0243] [INSPIRE].

[8] A. Brandhuber, P. Heslop and G. Travaglini, $M H V$ amplitudes in $N=4$ super Yang-Mills and Wilson loops, Nucl. Phys. B 794 (2008) 231 [arXiv:0707.1153] [inSPIRE].

[9] J.M. Drummond, J. Henn, G.P. Korchemsky and E. Sokatchev, On planar gluon amplitudes/Wilson loops duality, Nucl. Phys. B 795 (2008) 52 [arXiv:0709.2368] [InSPIRE].

[10] J.M. Drummond, J. Henn, G.P. Korchemsky and E. Sokatchev, Conformal Ward identities for Wilson loops and a test of the duality with gluon amplitudes, Nucl. Phys. B 826 (2010) 337 [arXiv:0712.1223] [INSPIRE].

[11] B. Basso, S. Komatsu and P. Vieira, Structure constants and integrable bootstrap in planar $N=4 S Y M$ theory, arXiv: 1505.06745 [INSPIRE].

[12] T. Fleury and S. Komatsu, Hexagonalization of correlation functions, JHEP 01 (2017) 130 [arXiv: 1611.05577] [INSPIRE].

[13] Z. Bern, L.J. Dixon and V.A. Smirnov, Iteration of planar amplitudes in maximally supersymmetric Yang-Mills theory at three loops and beyond, Phys. Rev. D 72 (2005) 085001 [hep-th/0505205] [INSPIRE].

[14] L.F. Alday and J. Maldacena, Comments on gluon scattering amplitudes via AdS/CFT, JHEP 11 (2007) 068 [arXiv:0710.1060] [INSPIRE].

[15] Z. Bern et al., The two-loop six-gluon MHV amplitude in maximally supersymmetric Yang-Mills theory, Phys. Rev. D 78 (2008) 045007 [arXiv:0803.1465] [InSPIRE].

[16] J.M. Drummond, J. Henn, G.P. Korchemsky and E. Sokatchev, Hexagon wilson loop = six-gluon MHV amplitude, Nucl. Phys. B 815 (2009) 142 [arXiv:0803.1466] [INSPIRE].

[17] A.B. Goncharov, M. Spradlin, C. Vergu and A. Volovich, Classical polylogarithms for amplitudes and Wilson loops, Phys. Rev. Lett. 105 (2010) 151605 [arXiv:1006.5703] [INSPIRE].

[18] V. Del Duca, C. Duhr and V.A. Smirnov, An analytic result for the two-loop hexagon Wilson loop in $N=4 S Y M$, JHEP 03 (2010) 099 [arXiv:0911.5332] [INSPIRE].

[19] L.J. Dixon, J.M. Drummond, M. von Hippel and J. Pennington, Hexagon functions and the three-loop remainder function, JHEP 12 (2013) 049 [arXiv:1308.2276] [INSPIRE].

[20] L.J. Dixon, J.M. Drummond, C. Duhr and J. Pennington, The four-loop remainder function and multi-Regge behavior at NNLLA in planar $N=4$ super-Yang-Mills theory, JHEP 06 (2014) 116 [arXiv:1402.3300] [INSPIRE].

[21] S. Caron-Huot, L.J. Dixon, A. McLeod and M. von Hippel, Bootstrapping a five-loop amplitude using Steinmann relations, Phys. Rev. Lett. 117 (2016) 241601 [arXiv: 1609.00669] [INSPIRE]. 
[22] J. Golden and M. Spradlin, An analytic result for the two-loop seven-point MHV amplitude in $\mathcal{N}=4$ SYM, JHEP 08 (2014) 154 [arXiv:1406.2055] [INSPIRE].

[23] V. Del Duca, C. Duhr and V.A. Smirnov, A two-loop octagon Wilson loop in $N=4 S Y M$, JHEP 09 (2010) 015 [arXiv: 1006.4127] [InSPIRE].

[24] P. Heslop and V.V. Khoze, Analytic results for MHV Wilson loops, JHEP 11 (2010) 035 [arXiv: 1007.1805] [INSPIRE].

[25] M. Kruczenski, A note on twist two operators in $N=4 S Y M$ and Wilson loops in Minkowski signature, JHEP 12 (2002) 024 [hep-th/0210115] [INSPIRE].

[26] K. Sakai and Y. Satoh, A Note on string solutions in $A d S_{3}$, JHEP 10 (2009) 001 [arXiv: 0907.5259] [INSPIRE].

[27] K. Sakai and Y. Satoh, Constant mean curvature surfaces in AdS $S_{3}$, JHEP 03 (2010) 077 [arXiv: 1001.1553] [INSPIRE].

[28] B. Basso, S. Caron-Huot and A. Sever, Adjoint BFKL at finite coupling: a short-cut from the collinear limit, JHEP 01 (2015) 027 [arXiv: 1407.3766] [INSPIRE].

[29] L.F. Alday and J. Maldacena, Null polygonal Wilson loops and minimal surfaces in Anti-de-Sitter space, JHEP 11 (2009) 082 [arXiv:0904.0663] [INSPIRE].

[30] L.F. Alday, D. Gaiotto and J. Maldacena, Thermodynamic bubble ansatz, JHEP 09 (2011) 032 [arXiv: 0911.4708] [INSPIRE].

[31] L.F. Alday, J. Maldacena, A. Sever and P. Vieira, Y-system for scattering amplitudes, J. Phys. A 43 (2010) 485401 [arXiv: 1002.2459] [INSPIRE].

[32] Y. Hatsuda, K. Ito, K. Sakai and Y. Satoh, Thermodynamic Bethe ansatz equations for minimal surfaces in $A d S_{3}$, JHEP 04 (2010) 108 [arXiv: 1002.2941] [INSPIRE].

[33] R. Koberle and J.A. Swieca, Factorizable Z(N) models, Phys. Lett. 86B (1979) 209 [INSPIRE].

[34] C.R. Fernandez-Pousa, M.V. Gallas, T.J. Hollowood and J.L. Miramontes, The symmetric space and homogeneous sine-Gordon theories, Nucl. Phys. B 484 (1997) 609 [hep-th/9606032] [INSPIRE].

[35] A.B. Zamolodchikov, Thermodynamic Bethe ansatz in relativistic models. scaling three state potts and Lee-Yang models, Nucl. Phys. B 342 (1990) 695 [INSPIRE].

[36] L.F. Alday et al., An operator product expansion for polygonal null Wilson loops, JHEP 04 (2011) 088 [arXiv: 1006.2788] [INSPIRE].

[37] Y. Hatsuda, K. Ito, K. Sakai and Y. Satoh, Six-point gluon scattering amplitudes from $Z_{4}$-symmetric integrable model, JHEP 09 (2010) 064 [arXiv:1005.4487] [INSPIRE].

[38] Y. Hatsuda, K. Ito, Y. Satoh and J. Suzuki, Quantum Wronskian approach to six-point gluon scattering amplitudes at strong coupling, JHEP 08 (2014) 162 [arXiv:1406.5904] [INSPIRE].

[39] Y. Hatsuda, K. Ito and Y. Satoh, Null-polygonal minimal surfaces in $A d S_{4}$ from perturbed $W$ minimal models, JHEP 02 (2013) 067 [arXiv:1211.6225] [INSPIRE].

[40] Y. Hatsuda, K. Ito, K. Sakai and Y. Satoh, g-functions and gluon scattering amplitudes at strong coupling, JHEP 04 (2011) 100 [arXiv:1102.2477] [INSPIRE].

[41] Y. Hatsuda, K. Ito and Y. Satoh, T-functions and multi-gluon scattering amplitudes, JHEP 02 (2012) 003 [arXiv:1109.5564] [INSPIRE].

[42] G. Jüttner, A. Klümper and J. Suzuki, Exact thermodynamics and Luttinger liquid properties of the integrable $t-J$ model, Nucl. Phys. B 487 (1997) 650 [InSPIRE]. 
[43] A.B. Zamolodchikov, Mass scale in the sine-Gordon model and its reductions, Int. J. Mod. Phys. A 10 (1995) 1125 [INSPIRE].

[44] A. Brandhuber, P. Heslop, V.V. Khoze and G. Travaglini, Simplicity of polygon Wilson loops in $N=4$ SYM, JHEP 01 (2010) 050 [arXiv:0910.4898] [INSPIRE].

[45] A.B. Zamolodchikov, On the thermodynamic Bethe ansatz equations for reflectionless ADE scattering theories, Phys. Lett. B 253 (1991) 391 [INSPIRE].

[46] S. Fomin and A. Zelevinsky, Cluster algebras IV. Coefficients, Compos. Math. 143 (2007) 112.

[47] R. Inoue et al., Periodicities of $T$ and $Y$-systems, dilogarithm identities, and cluster algebras I: type $B_{r}$, Publ. Res. Inst. Math. Sci. 49 (2013) 1.

[48] R. Inoue et al., Periodicities of $T$ and $Y$-systems, dilogarithm identities, and cluster algebras II: type $C_{r}, F_{4}$ and $G_{2}$, Publ. Res. Inst. Math. Sci. 49 (2013) 43.

[49] A. Kuniba, T. Nakanishi and J. Suzuki, T-systems and Y-systems in integrable systems, J. Phys. A 44 (2011) 103001 [arXiv:1010.1344] [InSPIRE].

[50] A. Sever and P. Vieira, Multichannel conformal blocks for polygon Wilson loops, JHEP 01 (2012) 070 [arXiv:1105.5748] [InSPIRE].

[51] D. Fioravanti, S. Piscaglia and M. Rossi, Asymptotic Bethe ansatz on the GKP vacuum as a defect spin chain: scattering, particles and minimal area Wilson loops, Nucl. Phys. B 898 (2015) 301 [arXiv: 1503.08795] [INSPIRE].

[52] A. Bonini, D. Fioravanti, S. Piscaglia and M. Rossi, Strong Wilson polygons from the lodge of free and bound mesons, JHEP 04 (2016) 029 [arXiv:1511.05851] [INSPIRE].

[53] J. Bartels, J. Kotanski and V. Schomerus, Excited hexagon Wilson loops for strongly coupled $N=4 S Y M, J H E P 01$ (2011) 096 [arXiv: 1009.3938] [INSPIRE].

[54] V.V. Bazhanov, S.L. Lukyanov and A.B. Zamolodchikov, Integrable structure of conformal field theory, quantum KdV theory and thermodynamic Bethe ansatz, Commun. Math. Phys. 177 (1996) 381 [hep-th/9412229] [INSPIRE].

[55] P. Dorey, I. Runkel, R. Tateo and G. Watts, $g$ function flow in perturbed boundary conformal field theories, Nucl. Phys. B 578 (2000) 85 [hep-th/9909216] [INSPIRE].

[56] P. Dorey, A. Lishman, C. Rim and R. Tateo, Reflection factors and exact g-functions for purely elastic scattering theories, Nucl. Phys. B 744 (2006) 239 [hep-th/0512337] [INSPIRE].

[57] V.A. Fateev, The exact relations between the coupling constants and the masses of particles for the integrable perturbed conformal field theories, Phys. Lett. B 324 (1994) 45 [InSPIRE].

[58] Z. Bajnok et al., Exact mass-coupling relation for the homogeneous sine-Gordon model, Phys. Rev. Lett. 116 (2016) 181601 [arXiv: 1512.04673] [INSPIRE].

[59] Z. Bajnok et al., On the mass-coupling relation of multi-scale quantum integrable models, JHEP 06 (2016) 071 [arXiv: 1604.02811] [INSPIRE]. 